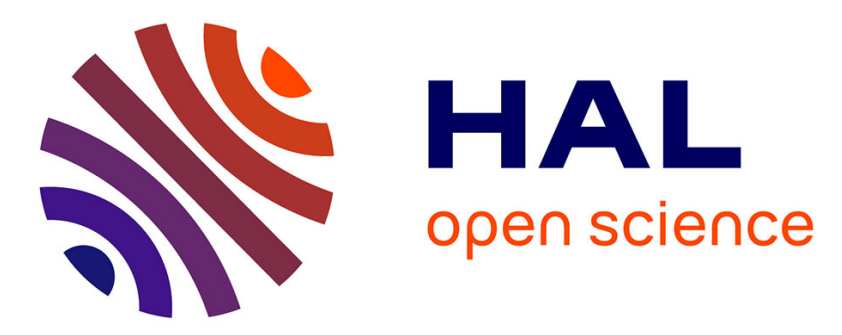

\title{
A comparison of edge-preserving approaches for differential interference contrast microscopy
}

Simone Rebegoldi, Lola Bautista, Laure Blanc-Féraud, Marco Prato, Luca Zanni, Arturo Plata

\section{To cite this version:}

Simone Rebegoldi, Lola Bautista, Laure Blanc-Féraud, Marco Prato, Luca Zanni, et al.. A comparison of edge-preserving approaches for differential interference contrast microscopy. Inverse Problems, 2017, 10.1088/1361-6420/aa790a . hal-01568695

\section{HAL Id: hal-01568695 \\ https://hal.inria.fr/hal-01568695}

Submitted on 25 Jul 2017

HAL is a multi-disciplinary open access archive for the deposit and dissemination of scientific research documents, whether they are published or not. The documents may come from teaching and research institutions in France or abroad, or from public or private research centers.
L'archive ouverte pluridisciplinaire HAL, est destinée au dépôt et à la diffusion de documents scientifiques de niveau recherche, publiés ou non, émanant des établissements d'enseignement et de recherche français ou étrangers, des laboratoires publics ou privés. 


\title{
A comparison of edge--preserving approaches for differential interference contrast microscopy
}

\author{
S Rebegoldi ${ }^{1}$, L Bautista ${ }^{2,3}$, L Blanc-Féraud ${ }^{3}$, M Prato ${ }^{1}$, L \\ Zanni $^{1}$ and A Plata ${ }^{2}$ \\ ${ }^{1}$ Dipartimento di Scienze Fisiche, Informatiche e Matematiche, Università di \\ Modena e Reggio Emilia, Via Campi 213/b, 41125 Modena, Italy \\ 2 Escuela de Ingeniería de Sistemas y Escuela de Física, Universidad Industrial de \\ Santander, 680002 Bucaramanga, Colombia \\ ${ }^{3}$ Université Côte d'Azur, CNRS, Laboratoire I3S, UMR 7271, 06903 Sophia \\ Antipolis, France \\ E-mail: simone.rebegoldi@unimore.it
}

\begin{abstract}
In this paper we address the problem of estimating the phase from color images acquired with differential-interference-contrast microscopy. In particular, we consider the nonlinear and nonconvex optimization problem obtained by regularizing a least-squares-like discrepancy term with an edge-preserving functional, given by either the hypersurface potential or the total variation one. We investigate the analytical properties of the resulting objective functions, proving the existence of minimum points, and we propose effective optimization tools able to obtain in both the smooth and the nonsmooth case accurate reconstructions with a reduced computational demand.
\end{abstract}

AMS classification scheme numbers: 65K05, 90C30, 90C90

Keywords: DIC microscopy, phase estimation, nonlinear optimization methods Submitted to: Inverse Problems

\section{Introduction}

Since their invention, microscopes have been a powerful tool in a variety of disciplines such as biology, medicine and the study of materials. In particular, the branch of optical microscopy (also referred as light microscopy) has been successfully applied in biomedical sciences and cell biology in order to study detailed structures and understand their function in biological specimens. The optical microscope uses visible light for illuminating the object and contains lenses that magnify the image of the object and focus the light on the retina of the observer's eye [1]. Optical microscopy includes several techniques, such as bright-field, dark-field, phase contrast, differential interference contrast 
(DIC), fluorescence and confocal microscopy. We refer to the work of Wilson and Bacic [2] for a comparison of the advantages and limitations of these techniques.

The technique of interest in this paper is DIC microscopy, designed by Allen, David and Nomarski [3] to overcome the inability to image unstained transparent biological specimens, which is typical of bright-field microscopes, while avoiding at the same time the halo artifacts of other techniques designed for the same purpose, such as phase contrast. DIC microscopes are able to provide contrast to images by exploiting the phase shifts in light induced by the transparent specimens (also called phase objects) while passing through them. This phenomenon is not detected by the human eye, neither by an automatic visual system, and occurs because of the interaction of light with different refractive indexes of both the specimen and its surrounding medium. In DIC microscopy, such phase shifts are converted into artificial black and white shadows in the image, which correspond to changes in the spatial gradient of the specimen's optical path length. Furthermore, this technique has been widely recognized by its possibility to use full numerical apertures in the objective, which results in high contrast images at high lateral resolution.

One disadvantage of DIC microscopy is that the observed images cannot be easily used for topographical and morphological interpretation, because the changes in phase of the light are hidden in the intensity image. It is then of vital importance to recover the specimen's phase function from the observed DIC images. The problem of phase estimation in optical imaging has been widely studied, as shown in the review made in [4]. Previous work for reconstructing the DIC phase function has been done by Munster et al [5], who retrieve the phase information by deconvolution with a Wiener filter; line integration of DIC images is proposed by Kam in [6], supposing that the line integration along the shear angle yields a positive definite image, which is not always the case since the intensity image is a nonlinear relation between the transmission function of the specimen and the point spread function of the microscope. Kou et al [7] introduced the use of transport of intensity equation to retrieve the phase function; Bostan et al [8] also used this approach, including a total variation regularization term to preserve the phase transitions. Finally, in the work of Preza $[9,10,11,12]$, the phase estimation in DIC microscopy has been addressed by considering the minimization of a Tikhonov regularized discrepancy term, which is performed by means of a modified nonlinear conjugate gradient $(\mathrm{CG})$ method.

In this work, we are interested in reconstructing the phase by minimization of a penalized least-squares (LS) term as proposed in [11], suitably generalized in order to extend the one color acquisition to polychromatic ones. Instead of a first order Tikhonov regularization, which tends to recover oversmoothed images, we consider two different penalties, the first one being the total variation (TV) functional which is suitable for piecewise constant images, while the second is the hypersurface (HS) potential [13], 
which is a smooth generalization of the TV able to reconstruct both sharp and smooth variations of the unknown phase. Since the latter choice leads to the minimization of a smooth functional, we consider a limited memory gradient method, in which suitable adaptive steplength parameters are chosen to improve the convergence rate of the algorithm. As concerns the TV-based model, we address the minimization problem by means of a recently proposed linesearch-based forward-backward method able to handle the nonsmoothness of the TV functional [14].

We performed different numerical tests on synthetic realistic images and we compared the proposed methods with both the original CG method proposed in [11], exploiting a gradient-free linesearch for the computation of the steplength parameter, and standard CG approaches. The results we obtained show that the performance of the limited memory gradient method in minimizing the LS+HS functional is much better than those of the CG approaches in terms of number of function/gradient evaluations and, therefore, computational time. Moreover, despite the difficulties due to the presence of a nondifferentiable term, also the linesearch-based forward-backward method proposed in the case of the TV functional is able to provide reconstructed images with a computational cost comparable to that of the gradient methods, thus leaving to a potential user freedom to choose the desired regularizer without losing in efficiency.

The organization of the paper is as follows. In Section 2, the DIC system for transmitted coherent light is described, together with the corresponding polychromatic image formation model. Furthermore the nonlinear inverse problem of the phase reconstruction and its corresponding optimization problem are presented, proving some analytical properties of the objective function, such as the existence of minimum points. In Section 3 the iterative optimization algorithms designed to address the phase reconstruction problem are detailed. In Section 4 numerical simulations on synthetic images are presented in order to evaluate efficiency and robustness of the considered approaches. Conclusions and perspectives are included in Section 5.

\section{Model and problem formulation}

\subsection{The DIC system}

DIC microscopy works under the principle of dual beam interference of polarized light, as depicted in Figure 1. Coherent light coming from a source is passed through a polarizer lens. Every incident ray of polarized light is splitted by a Nomarski prism placed at the front focal plane of the condenser. This splitting produces two wave components - ordinary and extraordinary - such that their corresponding electromagnetic fields are orthogonal and separated at a fixed shear distance $2 \Delta x$ along a specific shear direction, whose angle $\tau_{k}$ formed with the $x$-axis is denominated shear angle. The specimen is sampled by the pair of waves; if they pass through a region where there is a gradient 
in the refractive index, the waves will be differentially shifted in phase. After this, they will reach a second Normarski prism placed at the back focal plane of the objective lens. This prism introduces an additional phase shift, called the bias retardation and indicated with $2 \Delta \theta$, which helps to improve the contrast of the observed image and to give the shadow-cast effect characteristic of DIC images (see Figure 2). The interference of the two sheared and phase shifted waves occurs inside this prism and, thus, the two waves are recombined into a single beam that goes through a second polarizer lens called the analyzer. Further details on the DIC working principle can be found in the work of Murphy [15] and Mehta et al [16].

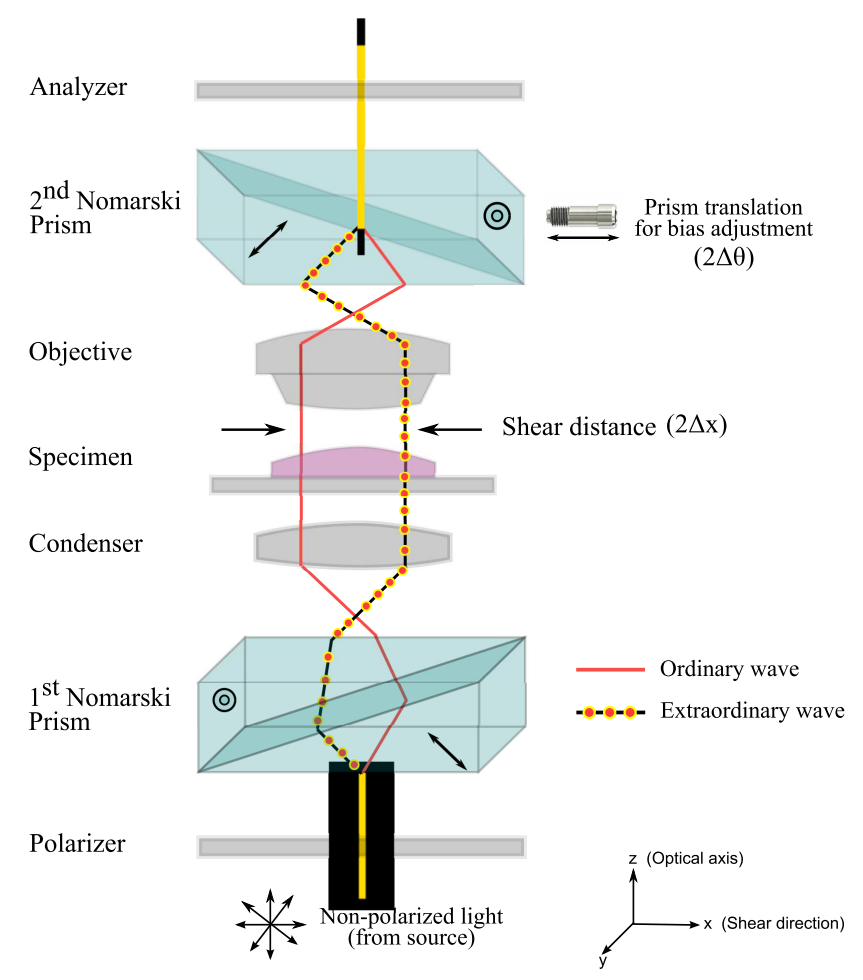

Figure 1. Transmitted light Nomarski DIC microscope. The difference of colors of the ordinary and extraordinary waves indicates that their electromagnetic fields are orthogonal to each other.

The observed images will have a uniform gray background on regions where there are no changes in the optical path, whereas they will have dark shadows and bright highlights where there are phase gradients in the direction of shear, having a 3D relieflike appearance (see Figure 2). It is important to note that the shadows and highlights indicate the signs and slope of phase gradients in the specimen, and not necessarily indicate high or low spots [3].

In this paper we consider the polychromatic rotational diversity model [17], which is an extension of the model presented in [11] to color image acquisition. In this model $K$ RGB color images are acquired by rotating the specimen $K$ times with respect to 


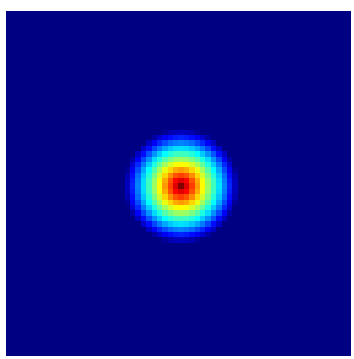

(a)

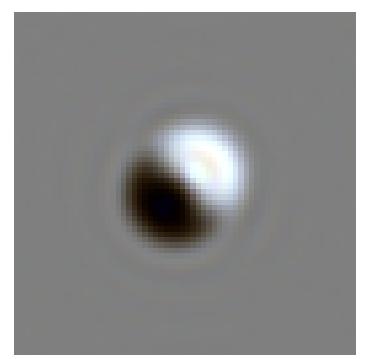

(b)

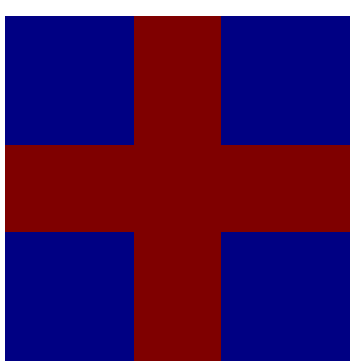

(c)

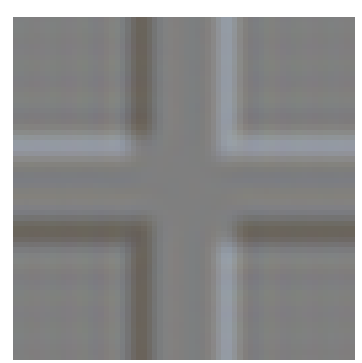

(d)

Figure 2. Phase functions of two phantom specimens and corresponding noiseless DIC color images: (a) phase function of the "cone" object, (b) DIC image of the cone, (c) phase function of the "cross" object, (d) DIC image of the cross. The images have been computed by using model (1) and setting the shear to $2 \Delta x=0.6 \mu \mathrm{m}$, the bias to $2 \Delta \theta=\pi / 2 \mathrm{rad}$ and the shear angle to $\tau=\pi / 4 \mathrm{rad}$.

the shear axis, which results in $K$ rotations of the amplitude point spread function. Typically $K$ equals 2 and the difference between the two angles is $\pi / 2$. Actually, for a given shear angle $\tau_{k}$, the acquired image $k$ is related to the directional derivative of the object along the direction $\tau_{k}$ [10]. Then the 2D image can be reconstructed from two orthogonal directional derivatives [11]. In this configuration, the relation between the acquired images and the unknown true phase $\phi$ is given by

$$
\left(o_{k, \lambda_{\ell}}\right)_{j}=a_{1}\left|\left(h_{k, \lambda_{\ell}} \otimes e^{-i \phi / \lambda_{\ell}}\right)_{j}\right|^{2}+\left(\eta_{k, \lambda_{\ell}}\right)_{j},
$$

for $k=1, \ldots, K, \ell=1,2,3, j \in \chi$, where

- $k$ is the index of the angles $\tau_{k}$ that the shear direction makes with the horizontal axis [11], $\ell$ is the index denoting one of the three RGB channels and $j=\left(j_{1}, j_{2}\right)$ is a $2 \mathrm{D}$ index varying in the set $\chi=\{1, \ldots, M\} \times\{1, \ldots, P\}, M$ and $P$ meaning the size of the acquired image, which is determined by the resolution of the CCD detector of the microscope, with typical value of $1388 \times 1040$ pixels;

- $\lambda_{\ell}$ is the $\ell-$ th illumination wavelength. The object is illuminated with white light, whose wavelengths range from $400 \mathrm{~nm}$ to $700 \mathrm{~nm}$. The digital acquisition system of the microscope comprises a color bandpass optical filter which isolates the RGB wavelengths, acquired separately by the CCD detector [15]. Since it is selected a narrow band for each color, we use the mean wavelength $\lambda_{\ell}$ at each band.

- $o_{k, \lambda_{\ell}} \in \mathbb{R}^{M P}$ is the $\ell^{t h}$ color component of the $k^{t h}$ discrete observed image $o_{k}=\left(o_{k, \lambda_{1}}, o_{k, \lambda_{2}}, o_{k, \lambda_{3}}\right) \in \mathbb{R}^{M P \times 3} ;$

- $\phi \in \mathbb{R}^{M P}$ is the unknown phase vector and $e^{-i \phi / \lambda_{\ell}} \in \mathbb{C}^{M P}$ stands for the vector defined by $\left(e^{-i \phi / \lambda_{\ell}}\right)_{j}=e^{-i \phi_{j} / \lambda_{\ell}}$;

- $h_{k, \lambda_{\ell}} \in \mathbb{C}^{M P}$ is the discretization of the continuous DIC point spread function $[10,18]$ corresponding to the illumination wavelength $\lambda_{\ell}$ and rotated by the angle $\tau_{k}$, i.e., $h_{k, \lambda_{\ell}}(x, y)=\frac{1}{2}\left[e^{-i \Delta \theta} p_{\lambda_{\ell}}\left(R_{k} \cdot(x-\Delta x, y)^{T}\right)-e^{i \Delta \theta} p_{\lambda_{\ell}}\left(R_{k} \cdot(x+\Delta x, y)^{T}\right)\right]$, 
where $p_{\lambda_{\ell}}(x, y)$ is the coherent PSF of the microscope's objective lens for the wavelength $\lambda_{\ell}$, which is given by the inverse Fourier transform of the disk support function of amplitude 1 and radius equal to the cutoff frequency $f_{c}=N A / \lambda_{\ell}$ [10], being $N A$ the numerical aperture of the objective lens, and $R_{k}$ is the rotation matrix which rotates the coordinates according to the shear angle $\tau_{k}$;

- $h_{1} \otimes h_{2}$ denotes the $2 \mathrm{D}$ convolution between the two $M \times P$ images $h_{1}, h_{2}$, extended with periodic boundary conditions;

- $\eta_{k, \lambda_{\ell}} \in \mathbb{R}^{M P}$ is the noise corrupting the data, which is a realization of a Gaussian random vector with mean $\mathbf{0} \in \mathbb{R}^{M P}$ and covariance matrix $\sigma^{2} I_{(M P)^{2}}$, where $I_{(M P)^{2}}$ is the identity matrix of size $(M P)^{2}$;

- $a_{1} \in \mathbb{R}$ is a constant which corresponds to closing the condenser aperture down to a single point.

\subsection{Least-squares data fidelity}

The phase reconstruction problem consists in finding an approximation of the unknown phase vector $\phi$ from the observed RGB images $o_{1}, \ldots, o_{K}$. Let us first address this problem by means of the maximum likelihood (ML) approach. Since the $3 K$ images $o_{k, \lambda_{\ell}}$ are corrupted by Gaussian noise, then the negative log likelihood of each image is a least-squares measure, which is nonlinear due to the presence of the exponential and the squared modulus in (1). In the case of white Gaussian noise, statistically independent of the data, the negative log likelihood of the problem is the sum of the negative log likelihoods of the different images, namely the following fit-to-data term

$$
J_{0}(\phi)=\sum_{\ell=1}^{3} \sum_{k=1}^{K} \sum_{j \in \chi}\left[\left(o_{k, \lambda_{\ell}}\right)_{j}-a_{1}\left|\left(h_{k, \lambda_{\ell}} \otimes e^{-i \phi / \lambda_{\ell}}\right)_{j}\right|^{2}\right]^{2} .
$$

Then the ML approach to the phase reconstruction inverse problem consists in the minimization of the function in (2):

$$
\min _{\phi \in \mathbb{R}^{M P}} J_{0}(\phi)
$$

In the next result, we collect some properties of $J_{0}$ that will be useful hereafter.

Lemma 1 Let $J_{0}: \mathbb{R}^{M P} \rightarrow \mathbb{R}$ be defined as in (2). The following properties hold true:

(i) $J_{0}(\phi+c \mathbf{1})=J_{0}(\phi), \forall c \in \mathbb{R}$, where $\mathbf{1} \in \mathbb{R}^{M P}$ is the vector of all ones.

(ii) $J_{0}$ is bounded.

(iii) $J_{0}$ is an analytic function on $\mathbb{R}^{M P}$ and therefore $J_{0} \in C^{\infty}\left(\mathbb{R}^{M P}\right)$.

(iv) Suppose that $\frac{\lambda_{\ell}}{\lambda_{\ell^{\prime}}}$ is rational for all $\ell, \ell^{\prime} \in\{1,2,3\}$. Then there exists $T>0$ such that $J_{0}$ is periodic of period $T$ with respect to each variable, i.e. for any $j \in \chi$, defining $\boldsymbol{e}_{j}=\left(\delta_{j, r}\right)_{r \in \chi}=(0, \ldots, 0,1,0, \ldots, 0) \in \mathbb{R}^{M P}$, where $\delta_{j, r}$ is the Kronecker delta, it holds

$$
J_{0}\left(\phi+T \boldsymbol{e}_{j}\right)=J_{0}(\phi), \quad \forall \phi \in \mathbb{R}^{M P} .
$$


Proof. (i) Set $J_{\ell, k, j}(\phi)=\left|\left(h_{k, \lambda_{\ell}} \otimes e^{-i \phi / \lambda_{\ell}}\right)_{j}\right|^{2}=\left|\sum_{r \in \chi}\left(h_{k, \lambda_{\ell}}\right)_{r} e^{-i\left(\phi_{j-r}\right) / \lambda_{\ell}}\right|^{2}$. If the thesis holds for $J_{\ell, k, j}$, then it holds also for $J_{0}$. We have

$J_{\ell, k, j}(\phi+c \mathbf{1})=\left|\sum_{r \in \chi}\left(h_{k, \lambda_{\ell}}\right)_{r} e^{-i\left(\phi_{j-r}+c\right) / \lambda_{\ell}}\right|^{2}=\left|e^{-i c / \lambda_{\ell}}\right|^{2}\left|\sum_{r \in \chi}\left(h_{k, \lambda_{\ell}}\right)_{r} e^{-i\left(\phi_{j-r}\right) / \lambda_{\ell}}\right|^{2}=J_{\ell, k, j}(\phi)$.

(ii) By applying the triangular inequality and the fact that $\left|e^{-i \phi_{j} / \lambda_{\ell}}\right|=1, j \in \chi$, we have

$$
\begin{aligned}
\left|J_{0}(\phi)\right| & \leq \sum_{\ell=1}^{3} \sum_{k=1}^{K} \sum_{j \in \chi}\left[\left|\left(o_{k, \lambda_{\ell}}\right)_{j}\right|+a_{1}\left(\sum_{r \in \chi}\left|\left(h_{k, \lambda_{\ell}}\right)_{r}\right|\left|e^{-i\left(\phi_{j-r}\right) / \lambda_{\ell}}\right|\right)^{2}\right]^{2} \\
& =\sum_{\ell=1}^{3} \sum_{k=1}^{K} \sum_{j \in \chi}\left[\left|\left(o_{k, \lambda_{\ell}}\right)_{j}\right|+a_{1}\left(\sum_{r \in \chi}\left|\left(h_{k, \lambda_{\ell}}\right)_{r}\right|\right)^{2}\right]^{2} .
\end{aligned}
$$

(iii) If $J_{\ell, k, j}$ is an analytic function on $\mathbb{R}^{M P}$, then $J_{0}$ is given by sums and compositions of analytic functions and thus it is itself analytic [19, Propositions 1.6.2 and 1.6.7]. Hence we focus on $J_{\ell, k, j}$. Since $\left(h_{k, \lambda_{\ell}}\right)_{r} \in \mathbb{C}$, it can be expressed in its trigonometric form $\left(h_{k, \lambda_{\ell}}\right)_{r}=\rho_{r} e^{i \theta_{r}}$, with $\rho_{r} \in \mathbb{R}_{\geq 0}, \theta_{r} \in[0,2 \pi)$. Then we can rewrite $J_{\ell, k, j}$ as follows

$$
J_{\ell, k, j}(\phi)=\left(\sum_{r \in \chi} \rho_{r} \cos \left(\theta_{r}-\left(\phi_{j-r} / \lambda_{\ell}\right)\right)\right)^{2}+\left(\sum_{r \in \chi} \rho_{r} \sin \left(\theta_{r}-\left(\phi_{j-r} / \lambda_{\ell}\right)\right)\right)^{2}
$$

and the thesis follows from the analyticity of the functions $\sin (\cdot), \cos (\cdot)$ and $(\cdot)^{2}$.

(iv) It is easy to see that $J_{0}$ is the sum of three periodic functions of variable $\phi_{j}$ whose periods are $2 \pi \lambda_{1}, 2 \pi \lambda_{2}$ and $2 \pi \lambda_{3}$, respectively. By recalling that the sum of two periodic functions is periodic if the ratio of the periods is a rational number, we can conclude that $J_{0}$ is periodic.

\subsection{Introducing regularization}

When the assumption of rationality on the wavelengths ratios holds, using points (iii) and (iv) of Lemma 1, it is easy to see that the solution to problem (3) exists. However, points (i) and (iv) imply that the solution is not unique and may be determined only up to an unknown real constant or to multiples of the period $T$ w.r.t. any variable $\phi_{j}$. Furthermore, $J_{0}$ is a nonconvex function of the phase $\phi$, thus it may admit several local minima as well as saddle points. In the light of these considerations, we can conclude that (3) is a severely ill-posed problem, which requires regularization in order to impose some a priori knowledge on the unknown phase. In particular, we propose to solve the following regularized optimization problem

$$
\min _{\phi \in \mathbb{R}^{M P}} J(\phi) \equiv J_{0}(\phi)+J_{R}(\phi),
$$


where $J_{0}$ is the least-squares (LS) distance defined in (2) and $J_{R}$ is a regularization functional. In particular, we consider hypersurface (HS) potential defined as [20, 21]

$$
J_{R}(\phi)=\mu \sum_{j \in \chi} \sqrt{\left((\mathcal{D} \phi)_{j}\right)_{1}^{2}+\left((\mathcal{D} \phi)_{j}\right)_{2}^{2}+\delta^{2}}
$$

where $\mu>0$ is a regularization parameter, the discrete gradient operator $\mathcal{D}: \mathbb{R}^{M P} \longrightarrow$ $\mathbb{R}^{2 M P}$ is set through the standard finite difference with periodic boundary conditions

$(\mathcal{D} \phi)_{j_{1}, j_{2}}=\left(\begin{array}{c}\left((\mathcal{D} \phi)_{j_{1}, j_{2}}\right)_{1} \\ \left((\mathcal{D} \phi)_{j_{1}, j_{2}}\right)_{2}\end{array}\right)=\left(\begin{array}{c}\phi_{j_{1}+1, j_{2}}-\phi_{j_{1}, j_{2}} \\ \phi_{j_{1}, j_{2}+1}-\phi_{j_{1}, j_{2}}\end{array}\right), \phi_{M+1, j_{2}}=\phi_{1, j_{2}}, \phi_{j_{1}, P+1}=\phi_{j_{1}, 1}$

and the additional parameter $\delta \geq 0$ plays the role of a threshold for the gradient of the phase. The choice of this kind of regularization term instead of the first order Tikhonov one used e.g. in $[11,12]$ lies in the capability of the HS regularizer to behave both as a Tikhonov-like regularization in regions where the gradient assumes small values (w.r.t. $\delta$ ), and as an edge-preserving regularizer in regions where the gradient is very large, as it happens in the neighborhood of jumps in the values of the phase. Moreover, we will also consider the case in which the regularization term is given by the standard total variation (TV) functional [22], which is defined exactly as in (5) by setting $\delta=0$. We remark that, even if the HS term can be seen as a smoothed version of the TV one, their effect is quite different on the recovered images since, e.g., reconstructions provided by HS can be free of cartoon effects, typical of TV regularization. Furthermore, one should be careful to adopt appropriate minimization algorithms in both cases, since the use of a method designed for smooth optimization to minimize the LS + HS functional with a very small $\delta$ typically leads to severe numerical instability problems.

Problem (4) is still a difficult nonconvex optimization problem and, when $\delta=0$, it is also nondifferentiable. Some properties of the objective function $J$ are now reported.

Lemma 2 Let $J: \mathbb{R}^{M P} \rightarrow \mathbb{R}$ be defined as in (4). Then:

(i) $J(\phi+c \mathbf{1})=J(\phi), \forall c \in \mathbb{R}$.

(ii) If $\delta>0$, then $J \in C^{\infty}\left(\mathbb{R}^{M P}\right)$ and $\nabla J$ is Lipschitz continuous, namely there exists $L>0$ such that

$$
\|\nabla J(\phi)-\nabla J(\psi)\|_{2} \leq L\|\phi-\psi\|_{2}, \quad \forall \phi, \psi \in \mathbb{R}^{M P} .
$$

Proof. (i) It follows from the relation $(\mathcal{D}(\phi+c \mathbf{1}))_{j_{1}, j_{2}}=(\mathcal{D} \phi)_{j_{1}, j_{2}}$.

(ii) Point (iii) of Lemma 1 states that $J_{0} \in C^{\infty}\left(\mathbb{R}^{M P}\right)$ and the same property holds for $J_{R}$ when $\delta>0$, hence $J$ is the sum of two $C^{\infty}\left(\mathbb{R}^{M P}\right)$ functions. It is known that $\nabla J_{R}$ is $L_{R}$-Lipschitz continuous with $L_{R}=8 \mu / \delta^{2}$. We prove that also $\nabla J_{0}$ is Lipschitz continuous. If we introduce the residual image $r_{k, \lambda_{\ell}}=\left|\left(h_{k, \lambda_{\ell}} \otimes e^{-i \phi / \lambda_{\ell}}\right)\right|^{2}-o_{k, \lambda_{\ell}}$ and fix $s \in \chi$, the partial derivative of $J_{0}$ and the entries of the Hessian matrix $\nabla^{2} J_{0}$ are given 
by

$$
\begin{gathered}
\frac{\partial J_{0}(\phi)}{\partial \phi_{s}}=\sum_{\ell=1}^{3} \sum_{k=1}^{K} \sum_{j \in \chi} \frac{4}{\lambda_{\ell}}\left(r_{k, \lambda_{\ell}}\right)_{j} \operatorname{Im}\left\{e^{-i \phi_{s} / \lambda_{\ell}}\left(h_{k, \lambda_{\ell}}\right)_{j-s} \overline{\left(h_{k, \lambda_{\ell}} \otimes e^{-i \phi / \lambda_{\ell}}\right)_{j}}\right\}, \\
\frac{\partial^{2} J_{0}(\phi)}{\partial \phi_{t} \partial \phi_{s}}=4 \sum_{\ell=1}^{3} \sum_{k=1}^{K} \sum_{j \in \chi} \frac{2}{\lambda_{\ell}^{2}} \operatorname{Im}\left\{\vartheta_{s}\right\} \operatorname{Im}\left\{\vartheta_{t}\right\}+ \\
\frac{\left(r_{k, \lambda_{\ell}}\right)_{j}}{\lambda_{\ell}^{2}} \operatorname{Re}\left\{e^{i\left(\phi_{t}-\phi_{s}\right) / \lambda_{\ell}}\left(h_{k, \lambda_{\ell}}\right)_{j-s} \overline{\left(h_{k, \lambda_{\ell}}\right)_{j-t}}-\delta_{s, t} \vartheta_{s}\right\},
\end{gathered}
$$

where $\vartheta_{p}=e^{-i \phi_{p} / \lambda_{\ell}}\left(h_{k, \lambda_{\ell}}\right)_{j-p} \overline{\left(h_{k, \lambda_{\ell}} \otimes e^{\left.-i \phi / \lambda_{\ell}\right)_{j}}\right.}(p \in \chi)$ and $\operatorname{Re}(\cdot), \operatorname{Im}(\cdot)$ denote the real and imaginary parts of a complex number. Since $\left|\vartheta_{p}\right| \leq\left|\left(h_{k, \lambda_{\ell}}\right)_{j-p}\right| \sum_{r \in \chi}\left|\left(h_{k, \lambda_{\ell}}\right)_{r}\right|$, we have

$$
\begin{aligned}
\left|\frac{\partial^{2} J_{0}(\phi)}{\partial \phi_{t} \partial \phi_{s}}\right| \leq 4 \sum_{\ell=1}^{3} \sum_{k=1}^{K} & \sum_{j \in \chi} \frac{2}{\lambda_{\ell}^{2}}\left|\left(h_{k, \lambda_{\ell}}\right)_{j-s}\right|\left|\left(h_{k, \lambda_{\ell}}\right)_{j-t}\right|\left(\sum_{r \in \chi}\left|\left(h_{k, \lambda_{\ell}}\right)_{r}\right|\right)^{2}+ \\
& \frac{\left|\left(r_{k, \lambda_{\ell}}\right)_{j}\right|}{\lambda_{\ell}^{2}}\left\{\left|\left(h_{k, \lambda_{\ell}}\right)_{j-s}\right|\left|\left(h_{k, \lambda_{\ell}}\right)_{j-t}\right|+\left|\left(h_{k, \lambda_{\ell}}\right)_{j-s}\right| \sum_{r \in \chi}\left|\left(h_{k, \lambda_{\ell}}\right)_{r}\right|\right\} .
\end{aligned}
$$

and therefore

$$
\begin{aligned}
& \left\|\nabla^{2} J_{0}(\phi)\right\|_{\infty}=\max _{t \in \chi} \sum_{s \in \chi}\left|\frac{\partial^{2} J_{0}(\phi)}{\partial \phi_{t} \partial \phi_{s}}\right| \\
& \leq 4 \sum_{\ell=1}^{3} \sum_{k=1}^{K} \sum_{j \in \chi} \frac{H_{k, \ell}}{\lambda_{\ell}^{2}}\left\{2 \max _{t \in \chi}\left|\left(h_{k, \lambda_{\ell}}\right)_{j-t}\right| H_{k, \ell}^{2}+\left(H_{k, \ell}^{2}+\left|\left(o_{k, \lambda_{\ell}}\right)_{j}\right|\right)\left[\max _{t \in \chi}\left|\left(h_{k, \lambda_{\ell}}\right)_{j-t}\right|+H_{k, \ell}\right]\right\} \\
& =L_{0}, \quad \forall \phi \in \mathbb{R}^{M P},
\end{aligned}
$$

where $H_{k, \ell}=\sum_{r \in \chi}\left|\left(h_{k, \lambda_{\ell}}\right)_{r}\right|$. From relation $\|A\|_{2} \leq \sqrt{\|A\|_{1}\|A\|_{\infty}}$ and the fact that $\left\|\nabla^{2} J(\phi)\right\|_{1}=\left\|\nabla^{2} J(\phi)\right\|_{\infty}\left(\nabla^{2} J_{0}(\phi)\right.$ is a symmetric matrix $)$, it follows that $\left\|\nabla^{2} J_{0}(\phi)\right\|_{2} \leq$ $L_{0}$ for all $\phi \in \mathbb{R}^{M P}$, which means that $\nabla J_{0}$ is $L_{0}$-Lipschitz continuous and consequently also $\nabla J$ is Lipschitz continuous with constant $L=L_{0}+L_{R}$.

Point (ii) of Lemma 2 shows that an estimate of the Lipschitz constant of $\nabla J_{0}$ and, in general, $\nabla J$ can be computed. The estimate derived in (8) is far from being sharp, but this will not affect the behaviour of the algorithms described in the next section since, unlike many existing proximal gradient or forward-backward methods which exploits the value of the Lipschitz constant of $\nabla J_{0}$, the methods we propose do not need it explicitly.

Point (i) of Lemma 2 makes clear that, if a solution to problem (4) exists, then it is not unique and it can be determined only up to a real constant. This is a common feature shared with the unregularized problem (3). However, since the objective function $J$ is not periodic and, in addition, none of the two terms $J_{0}$ and $J_{R}$ are coercive, we can not prove the existence of a minimum point of $J$ neither by continuity, as can be 
done for the function $J_{0}$ when the wavelengths ratios are rational, nor by coercivity. A specific proof of existence of the solution for problem (4) is now presented.

Theorem 1 The objective function $J$ admits at least one global minimum point. Furthermore, if $\psi \in \mathbb{R}^{M P}$ is a global minimizer of $J$, then also $\{\psi+c \mathbf{1}: c \in \mathbb{R}\}$ are global minimizers of $J$.

Proof. Let $S=\left\{\phi \in \mathbb{R}^{M P}: \phi=c \mathbf{1}, c \in \mathbb{R}\right\}$ be the line in $\mathbb{R}^{M P}$ of all constant images and $\Pi$ be any hyperplane intersecting $S$ in one point $\phi_{S}$, i.e.

$$
\Pi=\left\{\phi \in \mathbb{R}^{M P}: \sum_{r \in \chi} a_{r} \phi_{r}+b=0\right\}, \sum_{r \in \chi} a_{r} \neq 0, b \in \mathbb{R} .
$$

Thanks to part (i) of Lemma 2, for any $\phi \in \mathbb{R}^{M P}$ the point $\phi_{\Pi}=\phi-\left(\frac{\sum_{r} a_{r} \phi_{r}+b}{\sum_{r} a_{r}}\right) \mathbf{1} \in \Pi$ is such that $J\left(\phi_{\Pi}\right)=J(\phi)$. Consequently, if $\psi$ is a minimum point of $J$ on $\Pi$, then it is also a minimum point on $\mathbb{R}^{M P}$, because $J(\psi) \leq J\left(\phi_{\Pi}\right)=J(\phi)$ for all $\phi \in \mathbb{R}^{M P}$. Hence we restrict the search of the minimum point on $\Pi$ and we denote with $\left.J\right|_{\Pi}$ the restriction of $J$ to $\Pi$. Since $S=\arg \min _{\phi \in \mathbb{R}^{M P}} J_{R}(\phi)$ and $\Pi$ intersects $S$ only in $\phi_{S}, J_{R}$ is a convex function with a unique minimum point on $\Pi$, which implies that $J_{R}$ is coercive on $\Pi$ (see [23, Proposition 3.2.5, Definition 3.2.6]). Furthermore, from point (ii) of Lemma 1, we know that $J_{0}$ is a bounded function on $\Pi$. Then $\left.J\right|_{\Pi}$ is the sum of a coercive term and a bounded one, therefore it is itself coercive. This allows to conclude that $J$ admits a minimum point on $\Pi$ and thus also on $\mathbb{R}^{M P}$. The second part of the thesis follows from Lemma 2, part (i).

Note that the above proof of existence holds also for the regularized DIC problem proposed in $[11,12]$, in which the first order Tikhonov regularizer used instead of the TV functional is also noncoercive.

\section{Optimization methods}

In previous works $[9,11,12]$, the problem of DIC phase reconstruction had been addressed with a nonlinear conjugate gradient method [24]. However, as we will see in Section 4, these methods require in practice several evaluations of the objective function and possibly its gradient in order to compute the linesearch parameter. What we propose instead is to tackle problem (4) with a gradient descent algorithm in the differentiable case $(\delta>0)$ and a proximal gradient method in the nondifferentiable case $(\delta=0)$. The key ingredients of both methods are the use of an Armijo linesearch at each iteration, which ensures convergence to a stationary point of problem (4), and a clever adaptive choice of the steplength in order to improve the speed of convergence.

For sake of simplicity, from now on each monochromatic image is treated as a vector in $\mathbb{R}^{N}$ (being $N=M P$ ) obtained by a lexicographic reordering of its pixels. 


\subsection{The limited memory steepest descent method}

We consider first the HS regularizer. In this case the objective function is differentiable and we exploit the limited memory steepest descent (LMSD) method proposed by Fletcher [25] and outlined in Algorithm 1. The LMSD method is a standard gradient method equipped with a monotone Armijo linesearch and variable steplengths approximating the inverse of some eigenvalues of the Hessian matrix $\nabla^{2} J\left(\phi^{(n)}\right)$ in order to improve the convergence speed. Unlike the classical Barzilai-Borwein (BB) rules [26] and their generalizations (see e.g. $[27,28,29])$ which try to approximate $\left(\nabla^{2} J\left(\phi^{(n)}\right)\right)^{-1}$ with a constant diagonal matrix, the idea proposed by Fletcher for quadratic objective functions is based on a Lanczos iterative process applied to approximate some eigenvalues of the Hessian matrix of the objective function. Some algebra shows that this can be practically performed without the explicit knowledge of the Hessian itself but exploiting only a set of back gradients and steplengths (see steps 6-10 of Algorithm 1). Generalization to nonquadratic functions can be obtained by computing the eigenvalues of the matrix $\widetilde{\Phi}$ in step 10 instead of $\Phi$ (we remark that for quadratic $J$ the two matrices coincide).

Some practical issues have to be addressed in the implementation of Algorithm 1:

- The first loop (step 1 to 5 ) builds a matrix

$$
G=\left[\begin{array}{lll}
\nabla J\left(\phi^{(n-m)}\right) & \nabla J\left(\phi^{(n-m+1)}\right) \ldots \nabla J\left(\phi^{(n-1)}\right)
\end{array}\right]
$$

of size $M P \times m$. The initial values for the first $m$ steplengths can be provided by the user (e.g. by computing the BB ones) or can be chosen with the same approach described in steps $6-10$ but with smaller matrices. For example, one can fix $\alpha_{0}^{(0)}$, compute $G=\nabla J\left(\phi^{(0)}\right)$ and use steps 6-10 to compute $\alpha_{1}^{(0)}$. At this point, defining $G=\left[\begin{array}{ll}\nabla J\left(\phi^{(0)}\right) & \nabla J\left(\phi^{(1)}\right)\end{array}\right]$ one can compute $\alpha_{2}^{(0)}$ and $\alpha_{3}^{(0)}$ and repeat the procedure until a whole set of $m$ back gradients is available.

- The same procedure can be adopted when step 10 provides only $m^{\prime}<m$ positive eigenvalues. In this case, all columns of $G$ are discarded, $G$ becomes the empty matrix and the algorithm proceeds with $m^{\prime}$ instead of $m$ until a whole set of $m$ back gradients is computed. If $m^{\prime}=0$, a set of $m$ "safeguard" steplengths, corresponding to the last set of $m$ positive steplengths values provided by step 10, is exploited for the next $m$ iterations.

- If $G^{T} G$ in step 7 is not positive definite, then the oldest gradient of $G$ is discarded and a new matrix $G^{T} G$ is computed. This step is repeated until $G^{T} G$ becomes positive definite.

- The stopping criterion can be chosen by the user and be related to the decrease of the objective function $J$ or its gradient norm, or to the distance between two successive iterates.

Concerning the computational costs of LMSD, the heaviest tasks at each iteration are the computation of $\nabla J\left(\phi^{(n)}\right)$ at step 1 and $J\left(\phi^{(n)}-\alpha_{n} \nabla J\left(\phi^{(n)}\right)\right)$ at step 2 . 
Algorithm 1 Limited memory steepest descent (LMSD) method

Choose $\rho, \omega \in(0,1), m \in \mathbb{N}_{>0}, \alpha_{0}^{(0)}, \ldots, \alpha_{m-1}^{(0)}>0, \phi^{(0)} \in \mathbb{R}^{N}$ and set $n=0$.

While True

For $l=1, \ldots, m$

1. Define $G(:, l)=\nabla J\left(\phi^{(n)}\right)$.

2. Compute the smallest non-negative integer $i_{n}$ such that $\alpha_{n}=\alpha_{n}^{(0)} \rho^{i_{n}}$ satisfies

$$
J\left(\phi^{(n)}-\alpha_{n} \nabla J\left(\phi^{(n)}\right)\right) \leq J\left(\phi^{(n)}\right)-\omega \alpha_{n}\left\|\nabla J\left(\phi^{(n)}\right)\right\|^{2} .
$$

3. Compute $\phi^{(n+1)}=\phi^{(n)}-\alpha_{n} \nabla J\left(\phi^{(n)}\right)$.

If "Stopping Criterion" is satisfied

4. Return

Else

5. Set $n=n+1$.

EndIf

EndFor

6. Define the $(m+1) \times m$ matrix $\Gamma=\left[\begin{array}{ccc}\alpha_{n-m}^{-1} & & \\ -\alpha_{n-m}^{-1} & \ddots & \\ & \ddots & \alpha_{n-1}^{-1} \\ & & -\alpha_{n-1}^{-1}\end{array}\right]$.

7. Compute the Cholesky factorization $R^{T} R$ of the $m \times m$ matrix $G^{T} G$.

8. Solve the linear system $R^{T} r=G^{T} \nabla J\left(\phi^{(n)}\right)$.

9. Define the $m \times m$ matrix $\Phi=[R, r] \Gamma R^{-1}$.

10. Compute the eigenvalues $\theta_{1}, \ldots, \theta_{m}$ of the symmetric and tridiagonal approximation $\widetilde{\Phi}$ of $\Phi$ defined as

$$
\widetilde{\Phi}=\operatorname{diag}(\Phi)+\operatorname{tril}(\Phi,-1)+\operatorname{tril}(\Phi,-1)^{T},
$$

being $\operatorname{diag}(\cdot)$ and tril $(\cdot,-1)$ the diagonal and the strictly lower triangular parts of a matrix.

11. Define $\alpha_{n+i-1}^{(0)}=1 / \theta_{i}, i=1, \ldots, m$.

EndWhile

Considering step 1 , we focus on $\nabla J_{0}$. As it is written in (6), due to the product between $e^{-i \phi_{s} / \lambda_{\ell}}$ and $\left(h_{k, \lambda_{\ell}}\right)_{j-s}, \nabla J_{0}$ can be performed with $\mathcal{O}\left(N^{2}\right)$ complexity; this is how the gradient is computed in [11]. However, if we take the sum over $j$ of the residuals into the argument of $\operatorname{Im}(\cdot)$, then we can conveniently rewrite (6) as

$\frac{\partial J_{0}(\phi)}{\partial \phi_{s}}=\sum_{\ell=1}^{3} \sum_{k=1}^{K} \frac{4}{\lambda_{\ell}} \operatorname{Im}\left\{\left(\left(r_{k, \lambda_{\ell}} *\left(\overline{h_{k, \lambda_{\ell}}} \otimes e^{i \phi / \lambda_{\ell}}\right)\right) \otimes \tilde{h}_{k, \lambda_{\ell}}\right)_{s} e^{-i \phi_{s} / \lambda_{\ell}}\right\}$,

where $h_{1}$. $* h_{2}$ denotes the componentwise product between two images $h_{1}, h_{2}$ and $\left(\tilde{h}_{k, \lambda_{\ell}}\right)_{j}=\left(h_{k, \lambda_{\ell}}\right)_{-j}$ for all $j \in \chi$. Then the heaviest operations in (11) are the two 
convolutions which, thanks to the assumption of periodic boundary conditions, can be performed with a FFT/IFFT pair $\left(\mathcal{O}(N \log N)\right.$ complexity). Hence, since $\nabla J_{R}$ has $\mathcal{O}(N)$ complexity, we can conclude that step 1 has an overall complexity of $\mathcal{O}(N \log N)$. Similarly, the function at step 2 is computed with complexity $\mathcal{O}(N \log N)$, due to the presence of one convolution inside the triple sum in (2).

From a practical point of view, we have already shown that the LMSD method is an effective tool for DIC imaging, especially if compared to more standard gradient methods equipped with the BB rules [17]. From a mathematical point of view, one can prove, in the same way as in [30], that every limit point of the sequence generated by Algorithm 1 is a stationary point for problem (4). In addition, the convergence of Algorithm 1 can be asserted whenever the objective function $J$ satisfies the KurdykaŁojasiewicz (KL) property [31,32] at each point of its domain. More precisely, as shown in a number of recent papers $[33,34,35]$, one can prove the convergence of a sequence $\left\{\phi^{(n)}\right\}_{n \in \mathbb{N}}$ to a limit point (if any exists) which is stationary for $J$ if the following three conditions are satisfied:

$(H 1) \quad \exists a>0: J\left(\phi^{(n+1)}\right)+a\left\|\phi^{(n+1)}-\phi^{(n)}\right\|^{2} \leq J\left(\phi^{(n)}\right)$

(H2) $\exists b>0:\left\|\nabla J\left(\phi^{(n+1)}\right)\right\| \leq b\left\|\phi^{(n+1)}-\phi^{(n)}\right\|$

(H3) $J$ satisfies the KL property.

This scheme applies to the LMSD method. First of all, condition (H3) is satisfied for the DIC functional defined in (4). Indeed $J_{0}$ is an analytic function (Lemma 1, part (iii)) and $J_{R}$ is a semialgebraic function, which means that its graph is defined by a finite sequence of polynomial equations and inequalities (see [36] for a definition). Hence $J$ is the sum of an analytic function and a semialgebraic one and for this reason it satisfies the KL property on $\mathbb{R}^{N}$ (see [36, p. 1769] and references therein). Conditions (H1)(H2) follows from step 2 and 3, combined with the fact that $\nabla J$ is Lipschitz continuous (Lemma 2, part (ii)), provided that the sequence of steplengths $\alpha_{n}^{(0)}$ defined at step 11 is bounded from above. Therefore we can state the following result:

Theorem 2 Let $J$ be defined as in (4), $\left\{\phi^{(n)}\right\}_{n \in \mathbb{N}}$ the sequence generated by Algorithm 1 and $\alpha_{n}^{(0)} \leq \alpha_{\max }$, where $\alpha_{\max }>0$. If $\phi^{*}$ is a limit point of $\left\{\phi^{(n)}\right\}_{n \in \mathbb{N}}$, then $\phi^{*}$ is a stationary point of $J$ and $\phi^{(n)}$ converges to $\phi^{*}$.

Proof. We start by proving condition (H1). Step 3 of Algorithm 1 can be rewritten in the following way:

$$
-\alpha_{n} \nabla J\left(\phi^{(n)}\right)=\phi^{(n+1)}-\phi^{(n)}
$$

from which we have

$$
\alpha_{n}\left\|\nabla J\left(\phi^{(n)}\right)\right\|^{2}=\frac{1}{\alpha_{n}}\left\|\phi^{(n+1)}-\phi^{(n)}\right\|^{2} .
$$

By substituting (13) in step 2 and since $\alpha_{n} \leq \alpha_{n}^{(0)} \leq \alpha_{\max }$, we obtain

$$
J\left(\phi^{(n+1)}\right) \leq J\left(\phi^{(n)}\right)-\frac{\omega}{\alpha_{n}}\left\|\phi^{(n+1)}-\phi^{(n)}\right\|^{2} \leq J\left(\phi^{(n)}\right)-\frac{\omega}{\alpha_{\max }}\left\|\phi^{(n+1)}-\phi^{(n)}\right\|^{2} .
$$


Algorithm 2 Inexact linesearch-based algorithm (ILA)

Choose $0<\alpha_{\min } \leq \alpha_{\max }, \rho, \omega \in(0,1), \gamma \in[0,1], \tau>0, \phi^{(0)} \in \mathbb{R}^{N}$ and set $n=0$.

While True

1. Set $\alpha_{n}=\max \left\{\min \left\{\alpha_{n}^{(0)}, \alpha_{\max }\right\}, \alpha_{\min }\right\}$, where $\alpha_{n}^{(0)}$ is chosen as in Algorithm 1 .

2. Let $h_{\gamma}^{(n)}, h^{(n)}$ and $\psi^{(n)}$ be defined as in (18)-(19). Compute $\tilde{\psi}^{(n)} \in \mathbb{R}^{N}$ such that

$$
h^{(n)}\left(\tilde{\psi}^{(n)}\right)-h^{(n)}\left(\psi^{(n)}\right) \leq-\tau h_{\gamma}^{(n)}\left(\tilde{\psi}^{(n)}\right) .
$$

3. Set $d^{(n)}=\tilde{\psi}^{(n)}-\phi^{(n)}$.

4. Compute the smallest non-negative integer $i_{n}$ such that $\lambda_{n}=\rho^{i_{n}}$ satisfies

$$
J\left(\phi^{(n)}+\lambda_{n} d^{(n)}\right) \leq J\left(\phi^{(n)}\right)+\omega \lambda_{n} h_{\gamma}^{(n)}\left(\tilde{\psi}^{(n)}\right) .
$$

5. Compute the new point as $\phi^{(n+1)}=\phi^{(n)}+\lambda_{n} d^{(n)}$.

If "Stopping Criterion" is satisfied

6. Return

Else

7. Set $n=n+1$.

EndIf

EndWhile

Then (H1) holds with $a=\omega / \alpha_{\max }$. Regarding condition (H2), we can rewrite again step 3 as:

$$
\nabla J\left(\phi^{(n)}\right)=\frac{1}{\alpha_{n}}\left(\phi^{(n)}-\phi^{(n+1)}\right) .
$$

Recall that the Lipschitz continuity of $\nabla J$ implies that there is $\alpha_{\min }>0$ such that the linesearch parameter $\alpha_{n} \geq \alpha_{\min }$ (see [14, Proposition 4.2] for a proof). Then

$$
\begin{aligned}
\left\|\nabla J\left(\phi^{(n+1)}\right)\right\| & \leq\left\|\nabla J\left(\phi^{(n+1)}\right)-\nabla J\left(\phi^{(n)}\right)\right\|+\left\|\nabla J\left(\phi^{(n)}\right)\right\| \\
& \leq L\left\|\phi^{(n+1)}-\phi^{(n)}\right\|+\frac{1}{\alpha_{n}}\left\|\phi^{(n+1)}-\phi^{(n)}\right\| \\
& \leq\left(L+\frac{1}{\alpha_{\min }}\right)\left\|\phi^{(n+1)}-\phi^{(n)}\right\| .
\end{aligned}
$$

This concludes the proof of $(H 2)$ with $b=L+1 / \alpha_{\min }$. The thesis follows from [33, Theorem 2.9].

\subsection{Inexact linesearch-based algorithm}

We now turn to the algorithm we used to address the nonsmooth TV functional. In particular, we considered a simplified version of a recently proposed proximal gradient method called VMILA (variable metric inexact linesearch algorithm) [14]. In its general 
form, this method exploits a variable metric in the (possibly inexact) computation of the proximal point at each iteration and a backtracking loop to satisfy an Armijo-like inequality. Effective variable metrics can be designed for specific objective functions by exploiting suitable decompositions of the gradient of the smooth part of the objective function itself $[30,37,38,39]$. However, since in the DIC problem the gradient of $J_{0}$ does not lead to a natural decomposition in the required form, in our tests we used the standard Euclidean distance (we will denote with ILA this simplified version of VMILA).

The main steps of ILA are detailed in Algorithm 2. At each iteration $n$, given the point $\phi^{(n)} \in \mathbb{R}^{N}$ and the parameters $\alpha_{n}>0, \gamma \in[0,1]$, we define the function

$h_{\gamma}^{(n)}(\phi)=\nabla J_{0}\left(\phi^{(n)}\right)^{T}\left(\phi-\phi^{(n)}\right)+\frac{\gamma}{2 \alpha_{n}}\left\|\phi-\phi^{(n)}\right\|^{2}+J_{R}(\phi)-J_{R}\left(\phi^{(n)}\right)$.

We observe that $h_{\gamma}^{(n)}$ is strongly convex for any $\gamma \in(0,1]$. By setting $h^{(n)}=h_{1}^{(n)}$ and $z^{(n)}=\phi^{(n)}-\alpha_{n} \nabla J_{0}\left(\phi^{(n)}\right)$, we define the unique proximal point

$$
\psi^{(n)}:=\operatorname{prox}_{\alpha_{n} J_{R}}\left(z^{(n)}\right)=\arg \min _{\phi \in \mathbb{R}^{N}} h^{(n)}(\phi) .
$$

In step 2 of Algorithm 2, an approximation $\tilde{\psi}^{(n)}$ of the proximal point $\psi^{(n)}$ is defined by means of condition (16). Such a point can be practically computed by remarking that $J_{R}$ can be written as

$$
J_{R}(\phi)=g(\mathcal{D} \phi), \quad g(t)=\mu \sum_{j=1}^{N}\left\|\left(\begin{array}{c}
t_{2 j-1} \\
t_{2 j}
\end{array}\right)\right\|, \quad t \in \mathbb{R}^{2 N} .
$$

Then considering the dual problem of (19)

$$
\max _{v \in \mathbb{R}^{2 N}} \Gamma^{(n)}(v),
$$

the dual function $\Gamma^{(n)}$ has the following form

$\Gamma^{(n)}(v)=-\frac{\left\|\alpha_{n} \mathcal{D}^{T} v-z^{(n)}\right\|^{2}}{2 \alpha_{n}}-g^{*}(v)-J_{R}\left(\phi^{(n)}\right)-\frac{\alpha_{n}}{2}\left\|\nabla J_{0}\left(\phi^{(n)}\right)\right\|^{2}+\frac{\left\|z^{(n)}\right\|^{2}}{2 \alpha_{n}}$

where $g^{*}$ is the convex conjugate of $g$, namely the indicator function of the set $\left(B_{0, \mu}^{2}\right)^{N}$, being $B_{0, \mu}^{2} \subset \mathbb{R}^{2}$ the 2 -dimensional Euclidean ball centered in 0 with radius $\mu$.

Condition (16) is fulfilled by any point $\tilde{\psi}^{(n)}=z^{(n)}-\alpha_{n} A^{T} v$ with $v \in \mathbb{R}^{2 N}$ satisfying [14]

$$
h^{(n)}\left(\tilde{\psi}^{(n)}\right) \leq \eta \Gamma^{(n)}(v), \quad \eta=1 /(1+\tau)
$$

Such a point can be found by applying an iterative method to problem (20) and using (22) as stopping criterion.

Similarly to LMSD, any limit point of the sequence generated by ILA is stationary for problem (4) [14, Theorem 4.1] and, under the assumption that a limit point exists, the convergence of ILA to such a point holds when $J$ satisfies the Kurdyka-Łojasiewicz property, the gradient of the smooth part $\nabla J_{0}$ is Lipschitz continuous and the proximal point $\tilde{\psi}^{(n)}$ is computed exactly [35]. Whether and when ILA converges if the proximal point is computed inexactly is still an open problem, therefore all we can say for Algorithm 2 applied to the DIC problem is that all its limit points are stationary. 


\section{Numerical experiments}

In this section we test the effectiveness of the algorithms previously described in some synthetic problems. All the numerical results have been obtained on a PC equipped with an INTEL Core i7 processor 2.60GHz with 8GB of RAM running Matlab R2013a with its standard settings. For each test we will report the number of function evaluations, the number of gradient evaluations and the computational time needed by each algorithm to provide the reconstructed phase. With this information the reader should be able to estimate the complexity of the different approaches independently of the environment in which the algorithms are implemented and run. The LMSD and ILA routines for the DIC problem together with an illustrative example can be downloaded at the webpage http://www.oasis.unimore.it/site/home/software.html.

\subsection{Comparison with state-of-the-art methods}

Since in the DIC problem the evaluation of the gradient $\nabla J$ is computational demanding and its nonlinearity w.r.t. $\alpha$ requires a new computation for each step of the backtracking loop, in $[9,11]$ a heuristic version of a nonlinear conjugate gradient (CG) is used exploiting a gradient-free linesearch based on a polynomial approximation method. Although this formulation has practical advantages, the resulting scheme is not guaranteed to converge, and in our tests we experienced very different behaviours w.r.t. to the choice of some initial parameters of the linesearch procedure. For this reason, we also implemented several standard CG methods [24, 40], namely the Fletcher-Reeves (FR), Polak-Ribière (PR), PR with nonnegative values $\left(\mathrm{PR}^{+}\right)$and $\mathrm{PR}$ constrained by the FR values (FR-PR) strategies [41]. For these algorithms, the global convergence is ensured by computing the steplength parameter by means of the strong Wolfe conditions [24, 41].

The evaluations of the optimization methods have been carried out on two phantom objects (see Figure 3), which have been computed by using the formula for the phase difference between two waves travelling through two different media

$$
\phi_{s}=2 \pi\left(n_{1}-n_{2}\right) t_{s},
$$

where $n_{1}$ and $n_{2}$ are the refractive indices of the object structure and the surrounding medium, respectively, and $t_{s}$ is the thickness of the object at pixel $s \in \chi$. The first phantom, denominated "cone" and reported at the top row of Figure 3, is a $64 \times 64$ phase function representing a truncated cone of radius $r=3.2 \mu \mathrm{m}$ with $n_{1}=1.33$, $n_{2}=1$ and maximum value $\phi_{\max }=1.57 \mathrm{rad}$ attained at the cone vertex. The "cross" phantom, shown at the bottom row of Figure 3, is another $64 \times 64$ phase function of two crossing bars, each one of width $5 \mu \mathrm{m}$, measuring $0.114 \mathrm{rad}$ inside the bars and 0 in the background. For both simulations, the DIC microscope parameters were set as follows:

- shear: $2 \Delta x=0.6 \mu \mathrm{m}$;

- bias: $2 \Delta \theta=\pi / 2 \mathrm{rad}$; 

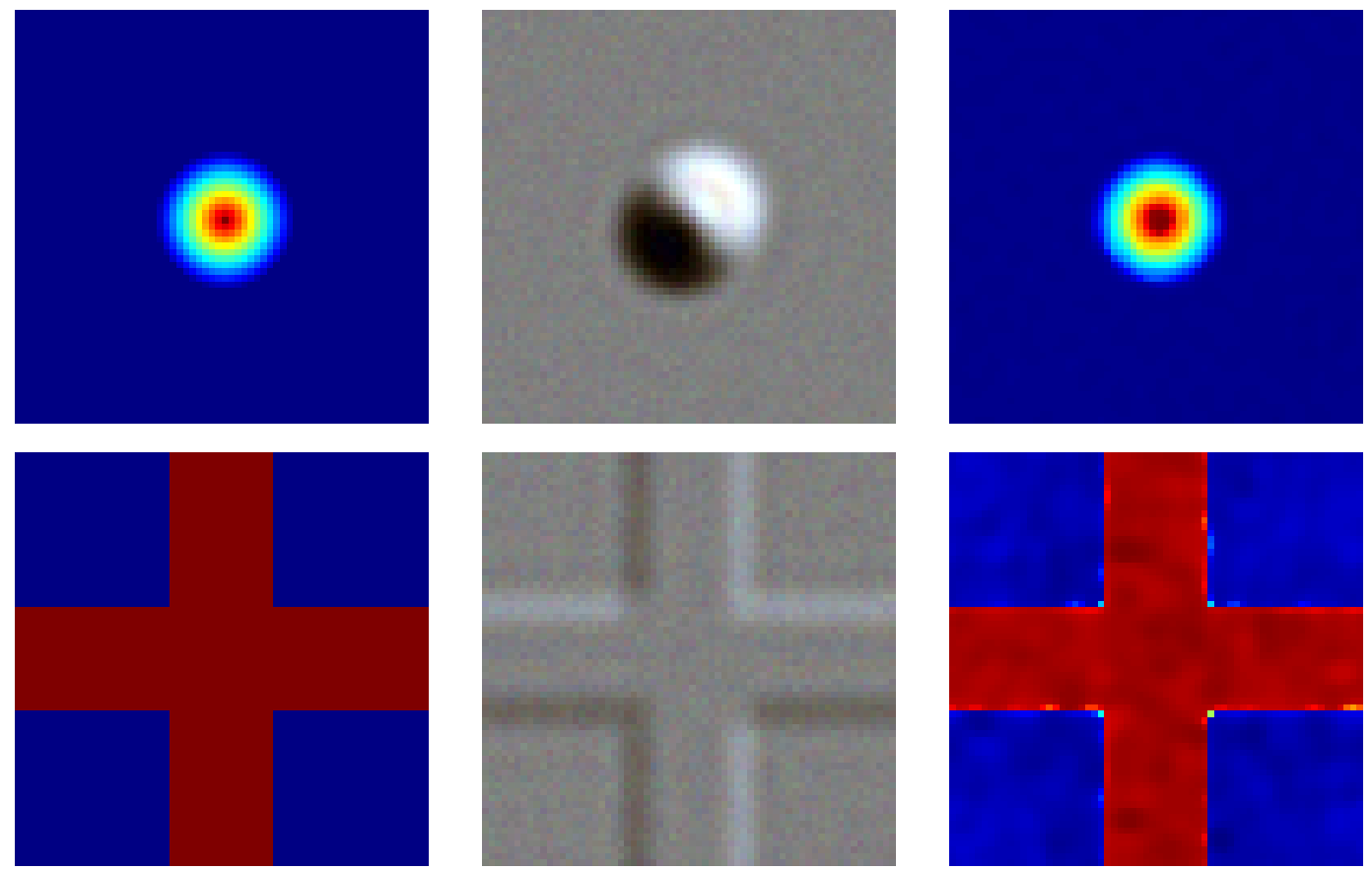

Figure 3. Data and results for the cone (top row) and cross (bottom row) objects. From left to right: true object, noisy DIC color image taken at shear angle $\frac{\pi}{4} \mathrm{rad}$ and corrupted with white Gaussian noise at $\mathrm{SNR}=4.5 \mathrm{~dB}$, and reconstructed phase with the LMSD method from observations at shear angles equal to $-\pi / 4 \mathrm{rad}$ and $\pi / 4 \mathrm{rad}$.

- numerical aperture of the objective: $\mathrm{NA}=0.9$.

For each phantom, a dataset consisting of $K=2$ polychromatic DIC images acquired at shear angles $\tau_{1}=-\pi / 4 \mathrm{rad}$ and $\tau_{2}=\pi / 4 \mathrm{rad}$ was created, as in model (1), by convolving the true phase function with the accordingly rotated DIC PSFs and then by corrupting the result with white Gaussian noise at different values of the signal-to-noise ratio

$$
\mathrm{SNR}=10 \log _{10}\left(\overline{\frac{\phi^{*}}{\sigma}}\right)
$$

where $\overline{\phi^{*}}$ is the mean value of the true object and $\sigma$ is the standard deviation of noise. The SNR values chosen in the simulations were $9 \mathrm{~dB}$ and $4.5 \mathrm{~dB}$.

As far as the regularization parameter $\mu$ and the threshold $\delta$ in (5) are concerned, these have been manually chosen from a fixed range in order to obtain a visually satisfactory reconstruction. Note that the parameters were first set in the differentiable case $(\delta>0)$ for the LMSD and the nonlinear CG methods and then the same value of the parameter $\mu$ was used also in the nondifferentiable case $(\delta=0)$ for the ILA method. The values reported below have been used for each simulation presented in this section. The resulting values have been $\mu=10^{-2}, \delta=10^{-2}$ for the cone and $\mu=4 \cdot 10^{-2}, \delta=10^{-3}$ for the cross. 
Some details regarding the choice of the parameters involved in the optimization methods of Section 3 are now provided. The linesearch parameters $\rho, \omega$ of the LMSD and ILA methods have been respectively set to $0.5,10^{-4}$. These are the standard choices for the Armijo parameters, however it is known that the linesearch algorithm is not so sensible to modifications of these values [30, 42]. The parameter $\gamma$ in the Armijo-like rule (17) has been fixed equal to 1, which corresponds to the mildest choice in terms of decrease of the objective function $J$. The parameter $m$ in Algorithm 1 is typically a small value $(m=3,4,5)$, in order to avoid a significant computational cost in the calculation of the steplengths $\alpha_{n}^{(0)}$; here we let $m=4$. The same choice for $m$ is done in Algorithm 2, where the values $\alpha_{n}^{(0)}$ are constrained in the interval $\left[\alpha_{\min }, \alpha_{\max }\right]$ with $\alpha_{\min }=10^{-5}$ and $\alpha_{\max }=10^{2}$. The dual problem (20) is addressed, at each iteration of ILA, by means of algorithm FISTA [43] which is stopped by using criterion (22) with $\eta=10^{-6}$. This value represents a good balance between convergence speed and computational time per iteration [14]. Concerning the nonlinear CG methods equipped with the strong Wolfe conditions, we use the same parameters as done in [41] and we initialize the related backtracking procedure as suggested in [24, p. 59]. Regarding the CG methods endowed with the polynomial approximation, a restart of the method is performed by taking a steepest descent step, whenever the search direction fails to be a descent direction. Finally, the constant phase object $\phi^{(0)}=0$ is chosen as initial guess for all methods.

In order to evaluate the performance of the phase reconstruction methods proposed in Section 3, we will make use of the following error distance

$$
E\left(\phi^{(n)}, \phi^{*}\right)=\min _{c \in \mathbb{R}} \frac{\left\|\phi^{(n)}-\phi^{*}-c \mathbf{1}\right\|}{\left\|\phi^{*}\right\|}=\frac{\left\|\phi^{(n)}-\phi^{*}-\bar{c} \mathbf{1}\right\|}{\left\|\phi^{*}\right\|}
$$

where $\phi^{*}$ is the phase to be reconstructed and $\bar{c}=\sum_{j \in \chi} \frac{\left(\phi_{j}^{(n)}-\phi_{j}^{*}\right)}{N}$. Unlike the usual root mean squared error, which is recovered by setting $c=0$ in (25), the error distance defined in $(25)$ is invariant with respect to phase shifts, i.e.

$$
E\left(\phi+c \mathbf{1}, \phi^{*}\right)=E\left(\phi, \phi^{*}\right), \quad \forall \phi \in \mathbb{R}^{N}, \forall c \in \mathbb{R} .
$$

That makes the choice of (25) well-suited for problem (4), whose solution might be recovered only up to a real constant.

The methods have been run for the cone and cross phantoms with the parameters setting previously outlined. Since in the unconstrained differentiable case the goal is to vanish of the gradient of $J$, the iterations of the LMSD and the CG methods have been arrested when the following stopping criterion based on the decrease of the gradient norm

$$
\left\|\nabla J\left(\phi^{(n)}\right)\right\| \leq \kappa
$$

was met with $\kappa=4 \cdot 10^{-2}$ for the cone and $\kappa=10^{-3}$ for the cross. On the other hand, since with the TV functional the gradient is not available, the ILA method has been 

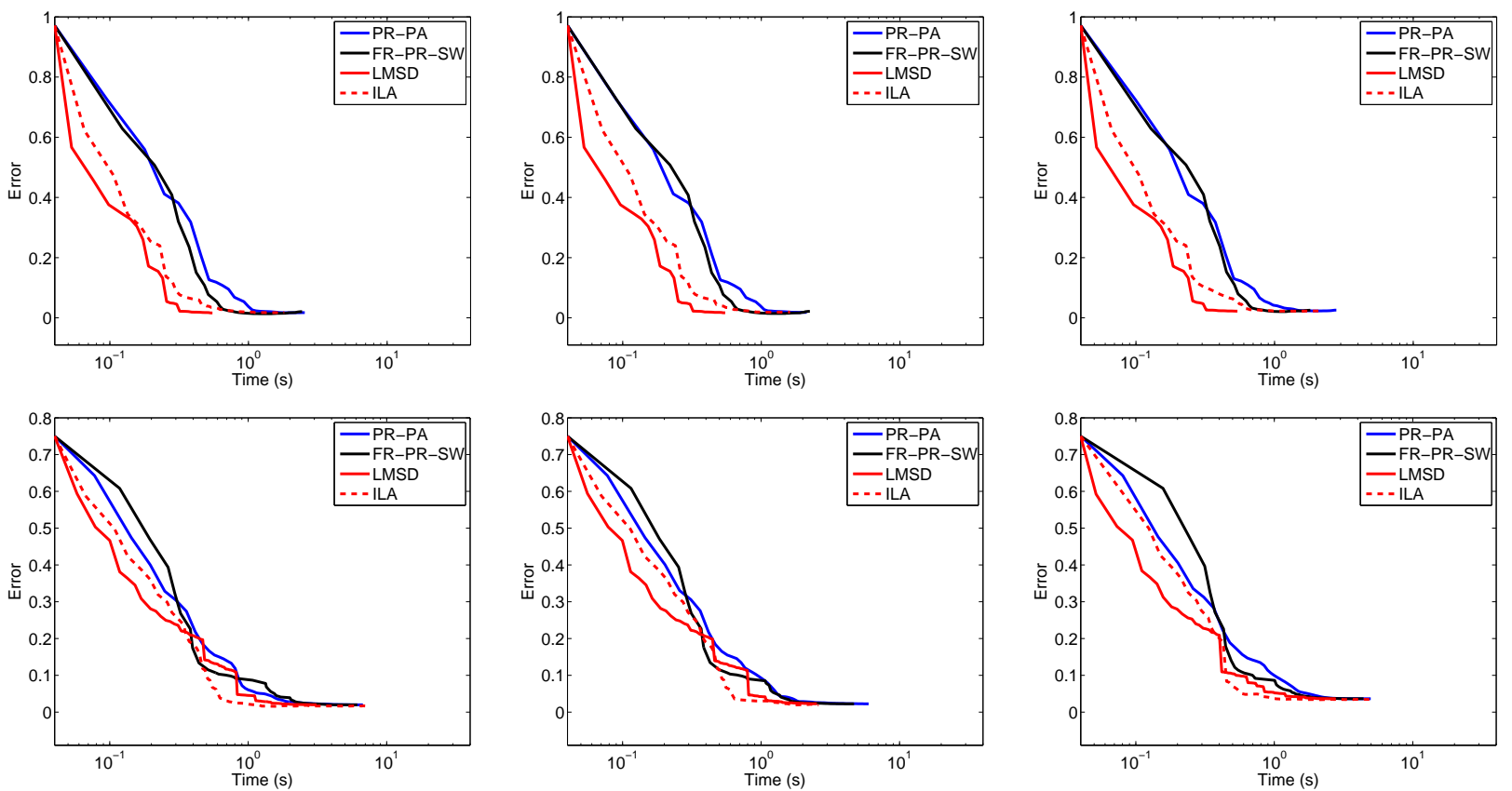

Figure 4. Error versus computational time plots for the cone (top row) and cross (bottom row) objects. From left to right: noise-free data, $\mathrm{SNR}=9 \mathrm{~dB}$ and $\mathrm{SNR}=$ $4.5 \mathrm{~dB}$.

stopped when the error up-to-a-constant between two successive iterates was lower than a prefixed $\kappa>0$, that is

$$
\frac{\left\|\phi^{(n+1)}-\phi^{(n)}-\left(\overline{\phi^{(n+1)}-\phi^{(n)}}\right) \mathbf{1}\right\|}{\left\|\phi^{(n+1)}\right\|} \leq \kappa,
$$

where $\overline{\phi^{(n+1)}-\phi^{(n)}}$ is the mean value of the difference between the two objects. The tolerance $\kappa$ in (28) was set equal to $5 \cdot 10^{-5}$ for the cone and $10^{-4}$ for the cross. We remark that these values, as the ones suggested before for the stopping criterion (27), have been tuned in order to obtain sensible reconstructions with errors close to the optimal ones.

In Figure 4 we show the reconstruction error provided by the different methods as a function of the computational time. Among the CG methods, we report only the results obtained by the $\mathrm{PR}$ algorithm combined with a polynomial-approximationbased linesearch (PR-PA) and the FR-PR one in which the linesearch parameter is computed with the SW conditions (FR-PR-SW), since they always outperformed the other possible choices. From the plots of Figure 4, it can be drawn that each method is quite stable with respect to the noise level on the DIC images. However, in terms of time efficiency, LMSD outperforms the CG methods in both tests, showing a time reduction of at least $50 \%$ to satisfy the stopping criterion. We report some further details in Tables 1 and 2 on the computational cost of the different methods. Of course the numerical values in the tables depend on the tolerances chosen for the stopping criteria, 


\begin{tabular}{cccccccc}
\hline SNR $(\mathrm{dB})$ & Algorithm & Iterations & $\# \mathrm{f}$ & $\# \mathrm{~g}$ & Time $(\mathrm{s})$ & Obj fun & Error \\
\hline \multirow{4}{*}{$\infty$} & PR-PA & 37 & 465 & 37 & 2.55 & 0.89 & $1.74 \%$ \\
& FR-PR-SW & 38 & 157 & 157 & 2.44 & 0.89 & $2.09 \%$ \\
& LMSD & 29 & 35 & 29 & 0.55 & 0.89 & $1.64 \%$ \\
& ILA & 66 & 119 & 66 & 1.77 & 0.52 & $1.76 \%$ \\
\hline \multirow{4}{*}{9} & PR-PA & 31 & 389 & 31 & 2.12 & 1.65 & $1.81 \%$ \\
& FR-PR-SW & 37 & 142 & 142 & 2.24 & 1.65 & $2.19 \%$ \\
& LMSD & 29 & 35 & 29 & 0.55 & 1.65 & $1.69 \%$ \\
4.5 & ILA & 60 & 91 & 60 & 1.56 & 1.29 & $1.91 \%$ \\
\hline \multirow{4}{*}{} & PR-PA & 41 & 514 & 41 & 2.79 & 6.88 & $2.57 \%$ \\
& FR-PR-SW & 34 & 115 & 115 & 1.81 & 6.88 & $2.54 \%$ \\
& LMSD & 29 & 35 & 29 & 0.54 & 6.88 & $2.22 \%$ \\
& ILA & 61 & 104 & 61 & 1.56 & 6.80 & $2.50 \%$ \\
\hline
\end{tabular}

Table 1. Cone tests. From left to right: number of iterations required to meet the stopping criteria, number of function and gradient evaluations, execution time, objective function value and error achieved at the last iteration.

but some general considerations can be drawn, e.g., on the number of evaluations of $J$ and $\nabla J\left(\nabla J_{0}\right.$ for ILA). For instance, in the case of the cone (Table 1), LMSD evaluates the function less than 2 times per iteration. By contrast, the backtracking procedure exploited in the FR-PR-SW method requires an average of 4 evaluations per iteration of both the function and gradient to satisfy the strong Wolfe conditions, whereas the PRPA method, despite evaluating the gradient only once, need on average 12 evaluations of the function before detecting the correct three-points-interval (see [11]). One could reduce the number of evaluations in $\mathrm{PR}-\mathrm{PA}$ by properly tuning the initial parameters of the linesearch. However, as mentioned before, this method is quite sensitive to this choice, and little variations might result in a great increase of the number of restarts and, eventually, in the divergence of the algorithm. In addition, it seems that the optimal value of these parameters strictly depends on the object to be reconstructed.

\subsection{Comparison between LMSD and ILA}

We now compare the performance of LMSD and ILA. On one hand, ILA reconstructs the cross object slightly better than LMSD. Indeed, ILA provides the lowest reconstruction error in Table 2 for each SNR value and the corresponding phase estimates have better preserved edges, as clearly depicted in Figure 5, where we consider the following "upto-a-constant" residual

$$
R_{j}=\left|\phi_{j}-\phi_{j}^{*}-\overline{\phi-\phi^{*}}\right|, \quad \forall j \in \chi
$$

to measure the quality of the reconstructions provided by the two methods. This result was expected, since ILA addresses problem (4) with the standard TV functional $(\delta=0$ in $(5))$, which is more suited than HS regularization $(\delta>0)$ when the object to be 


\begin{tabular}{cccccccc}
\hline SNR $(\mathrm{dB})$ & Algorithm & Iterations & $\# \mathrm{f}$ & $\# \mathrm{~g}$ & Time $(\mathrm{s})$ & Obj fun & Error \\
\hline \multirow{4}{*}{$\infty$} & PR-PA & 138 & 1373 & 138 & 6.73 & 1.01 & $1.98 \%$ \\
& FR-PR-SW & 109 & 423 & 423 & 6.14 & 1.01 & $1.98 \%$ \\
& LMSD & 168 & 231 & 168 & 3.09 & 1.01 & $2.00 \%$ \\
& ILA & 100 & 176 & 100 & 7.18 & 0.87 & $1.66 \%$ \\
\hline \multirow{4}{*}{9} & PR-PA & 121 & 1209 & 121 & 5.97 & 1.96 & $2.26 \%$ \\
& FR-PR-SW & 106 & 323 & 323 & 4.69 & 1.96 & $2.25 \%$ \\
& LMSD & 140 & 190 & 140 & 2.52 & 1.96 & $2.27 \%$ \\
4.5 & ILA & 57 & 106 & 57 & 2.60 & 1.82 & $1.94 \%$ \\
\hline \multirow{4}{*}{} & PR-PA & 98 & 997 & 98 & 4.97 & 8.57 & $3.63 \%$ \\
& FR-PR-SW & 96 & 300 & 300 & 4.41 & 8.57 & $3.63 \%$ \\
& LMSD & 152 & 221 & 152 & 2.75 & 8.57 & $3.64 \%$ \\
& ILA & 97 & 179 & 97 & 5.26 & 8.47 & $3.46 \%$ \\
\hline
\end{tabular}

Table 2. Cross tests. From left to right: number of iterations required to meet the stopping criteria, number of function and gradient evaluations, execution time, objective function value and error achieved at the last iteration.

reconstructed is piecewise constant. On the other hand, ILA may be computationally more expensive since, unlike LMSD, it requires to iteratively solve the inner subproblem (20) at each outer iteration. Indeed, looking at Table 2 we notice that, although the number of function evaluations per iteration in LMSD and ILA is quite similar (on average around 1.4 for LMSD and 1.8 for ILA) and the ILA iterations are stopped way before the LMSD ones, the computational time in ILA is always higher. For instance, in the case $\mathrm{SNR}=9 \mathrm{~dB}$, the methods require approximately the same time, although the number of iterations of ILA is more than halved. This fact is explained if we look at the average number of inner iterations required by ILA to compute the approximate proximal point: 21.3, 10.11 and 13.43 for $\mathrm{SNR}=\infty, 9,4.5 \mathrm{~dB}$ respectively. Analogous conclusions on the costs per iteration can be drawn by considering the results on the cone object (see Table 1). In this case, LMSD is able to achieve a lower reconstruction error w.r.t. ILA in very few iterations, providing a remarkable gain in the computational time needed.

In order to deepen the analysis between the differentiable TV approximation and the original nondifferentiable one, we compared the LMSD and ILA methods in one further realistic simulation. In particular, we considered the "grid" object in Figure 6, which is a $1388 \times 1040$ image emulating the phase function of a multi-area calibration artifact [44], which measures $1.212 \mathrm{rad}$ inside the black regions and $2.187 \mathrm{rad}$ inside the white ones. The setup of the two methods is identical to that of the previous tests (with the exception of the numerical aperture of the objective NA which has been set equal to 0.8 ), and the parameters $\mu$ (for both models) and $\delta$ (for the smooth TV functional) have been set equal to $2 \cdot 10^{-1}$ and $10^{-1}$, respectively. Instead of three levels of noise, 

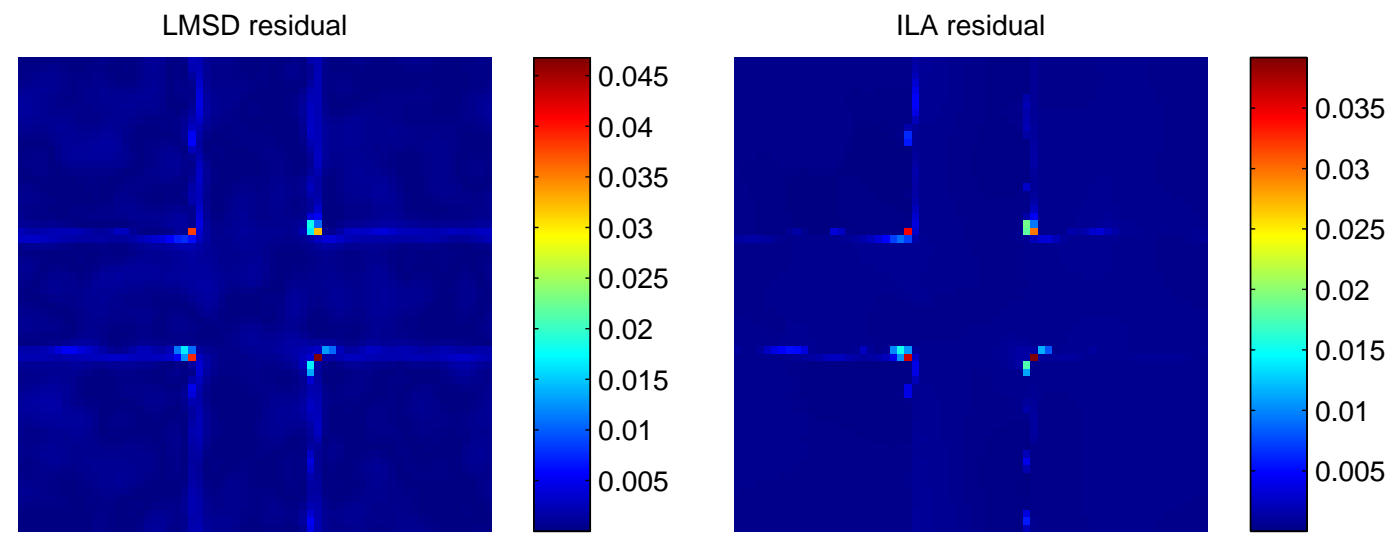

Figure 5. Cross test. The residuals defined in (29) for the reconstructions provided by LMSD and ILA, respectively, when the acquired images are corrupted with SNR = $9 \mathrm{~dB}$.
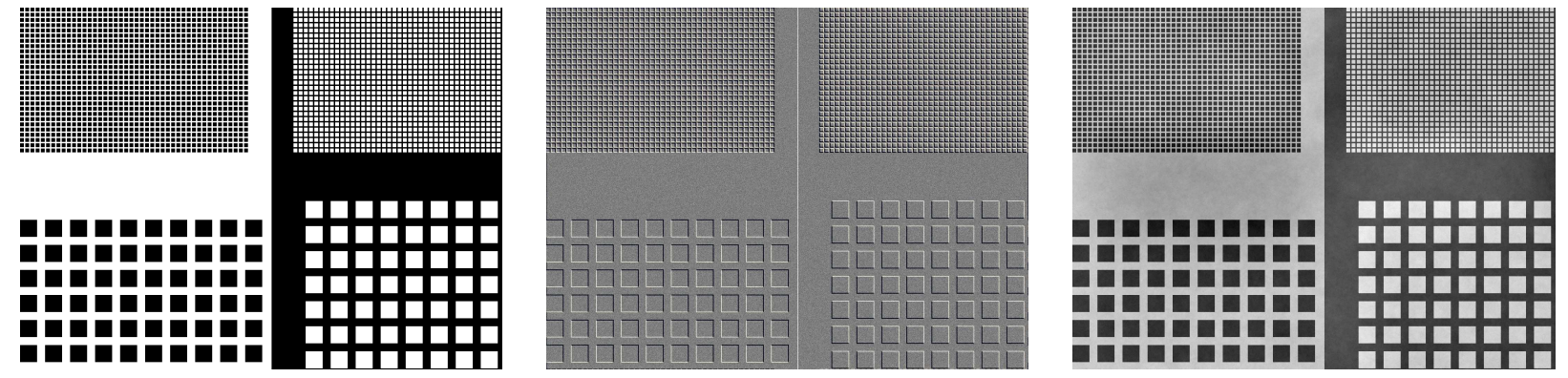

Figure 6. Data and results for the grid object. From left to right: true object, noisy DIC color image taken at shear angle $\frac{\pi}{4}$ rad and corrupted with white Gaussian noise at $\mathrm{SNR}=9 \mathrm{~dB}$, and reconstructed phase with the LMSD method from observations at shear angles equal to $-\pi / 4 \mathrm{rad}$ and $\pi / 4 \mathrm{rad}$.

here we only considered a SNR equal to $9 \mathrm{~dB}$. In Figure 7 we report the behaviour of the error (25) as a function of time and the number of inner iterations needed by ILA to address problem (20)-(22).

The grid dataset confirms the remarks previously done, since ILA takes almost twice as long compared to LMSD to provide an estimate of the phase. This is again due to the number of inner iterations, which starts to oscillatory increase after the first 20 iterations (see Figure 7). To conclude, we reckon that the LMSD method is generally preferable since, unlike ILA, it does not require any inner subproblem to be solved and thus it is generally less expensive from the computational point of view. However, the ILA method should be considered as a valid alternative when the object to be reconstructed is piecewise constant. 

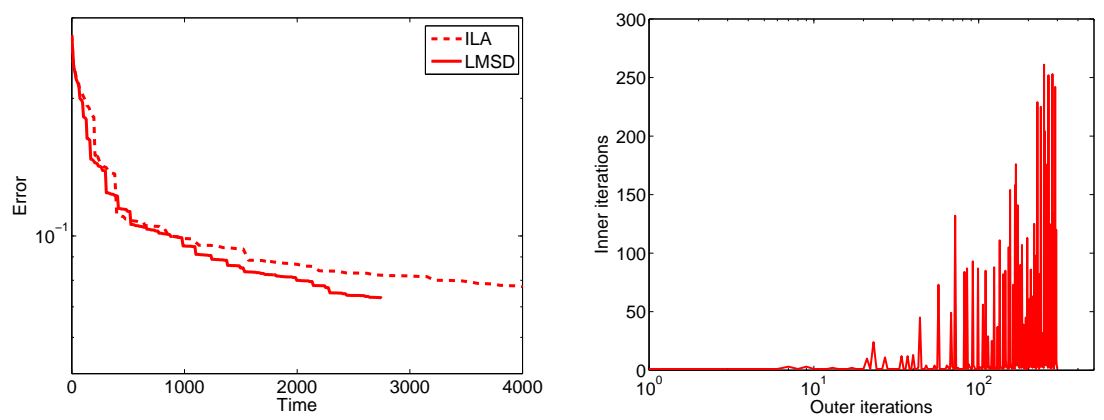

Figure 7. Grid test. From left to right: error versus time plots for LMSD and ILA and number of inner iterations versus number of outer iterations for ILA.

\subsection{Influence of color and bias retardation on phase reconstruction}

Another analysis of our interest was to observe how color information and bias retardation in the observations affect the behavior of phase reconstruction. We set four scenarios for comparison: independent monochromatic observations with red, green, and blue light, and polychromatic observation where all wavelengths are combined. For each of these scenarios we used the cross object to generate 100 observations at different realizations of noise, for both $\mathrm{SNR}=4.5 \mathrm{~dB}$ and $\mathrm{SNR}=9 \mathrm{~dB}$, and bias retardation of 0 $\mathrm{rad}$ and $\pi / 2 \mathrm{rad}$, at shear angles equal to $-\pi / 4 \mathrm{rad}$ and $\pi / 4 \mathrm{rad}$. We tested the LMSD method to perform the reconstructions; results for $\mathrm{SNR}=4.5 \mathrm{~dB}$ are shown in Figure 8 and for $\mathrm{SNR}=9 \mathrm{~dB}$ in Figure 9.
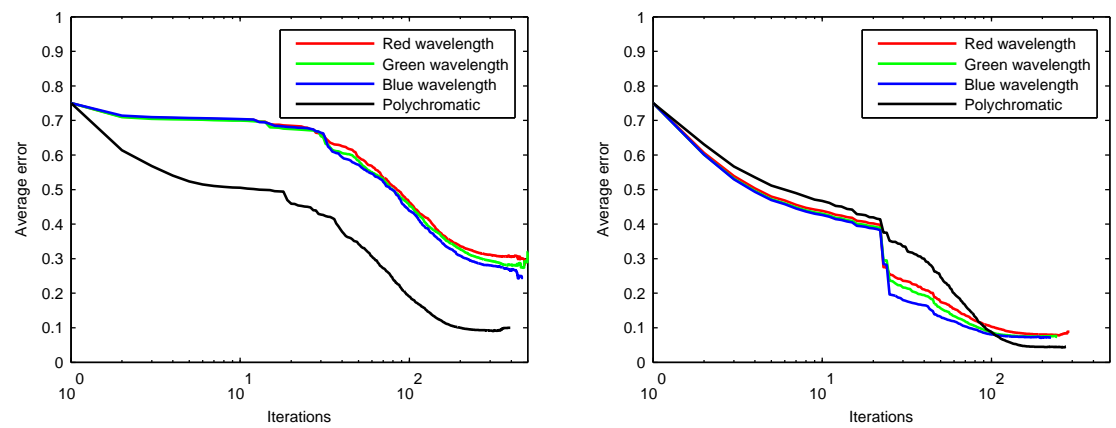

Figure 8. Average error comparison between monochromatic and polychromatic reconstructions. $\mathrm{SNR}=4.5 \mathrm{~dB}$. Left: bias $0 \mathrm{rad}$; right: bias $\pi / 2 \mathrm{rad}$.

The lines show the average error over the 100 observations. It is noticed that for 0 rad bias retardation, the reconstruction for polychromatic observations behave better than for the monochromatic ones, even though the amount of error is not promising of a good reconstruction. For $\pi / 2 \mathrm{rad}$ bias retardation the algorithm stops before the maximum number of iterations (500) is reached. In this case, for both levels of noise, the performance of the reconstruction with polychromatic light is quite comparable with monochromatic light. Another interesting finding about the convergence for 

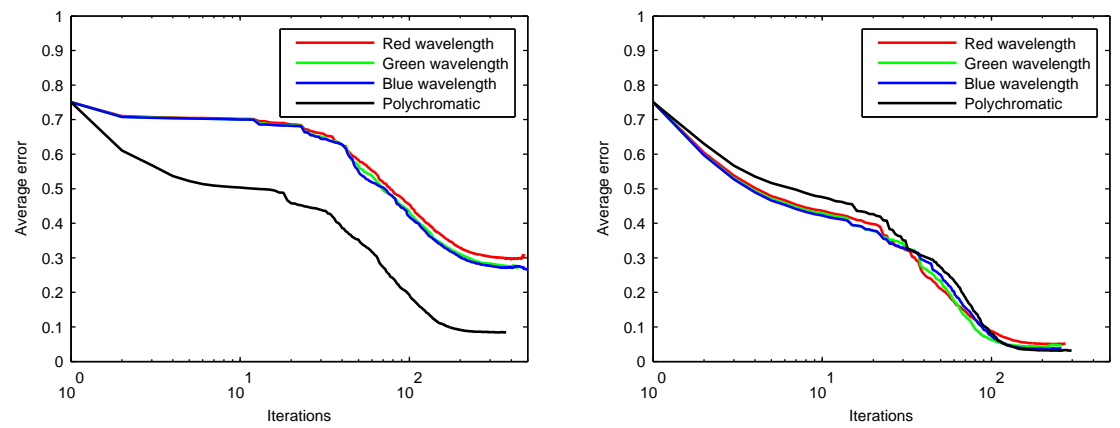

Figure 9. Average error comparison between monochromatic and polychromatic reconstructions. SNR $=9 \mathrm{~dB}$. Left: bias $0 \mathrm{rad}$; right: bias $\pi / 2 \mathrm{rad}$.

monochromatic light, is that for all cases, it happens in the order red-green-blue; this is due to the fact that the amplitude PSF for blue light has the bigger frequency support, thus provides more information for reconstruction.

\section{Conclusions and future work}

In this paper we provided both theoretical and practical contributions to the inverse problem of phase estimation from polychromatic DIC images. First of all, we studied the analytical properties of the LS data fidelity function arising from a maximum likelihood approach, showing its periodicity, shift-invariance and analyticity. Secondly, we analyzed the minimization problem of the functional given by the sum of the discrepancy term and an edge-preserving regularizer, proving the existence of minimum points. Finally, we proposed two recent optimization strategies for the case of both smooth and nonsmooth regularizers, and compared their performance with state-of-the-art conjugate gradient methods. In particular, for the HS regularizer we considered the LMSD method while in the case of the nonsmooth TV functional we proposed to exploit the ILA approach.

From the analysis we performed we drive the conclusions that an edge-preserving regularizer combined with an effective optimization tool can rapidly provide a good reconstruction of the phase. Of course the LMSD method has a much simpler structure than ILA and, in general, it should converge faster since ILA depends on two cycles of iterations (the outer defining the sequence and the inner computing the proximal point). However, in our tests the differences in time are not so significant, therefore a possible user might prefer to avoid the choice of a further parameter (the $\delta$ defining the HS term) and adopt the standard LS+TV model.

Future work will concern the application of the proposed strategies to real acquisitions and the reformulation of the minimization problem in terms of the specimen's transmission function $e^{-i \phi}$. This would lead to a standard regularized 
least-squares problem restricted to a nonconvex feasible set, which would require a generalization of the (VM)ILA approach able to account for nonconvex projections and to exploit the steplength selection rule proposed by Fletcher [25] in the presence of constraints $[45,46]$.

\section{Acknowledgments}

We would like to thank the anonymous reviewers for their valuable remarks and suggestions. This work has been partially supported by the ECOS-Nord grant C15M01, the Universidad Industrial de Santander and the Italian Ministry for University and Research (MIUR) under the projects FIRB - Futuro in Ricerca 2012, contract RBFR12M3AC, and PRIN 2012, contract 2012MTE38N. The Italian GNCS-INdAM (Gruppo Nazionale per il Calcolo Scientifico - Istituto Nazionale di Alta Matematica) is also acknowledged.

\section{References}

[1] S. P. Singh and B. S. Tomar. Cell Biology. Rastogi Publications, India, 2007.

[2] S. M. Wilson and A. Bacic. Preparation of plant cells for transmission electron microscopy to optimize immunogold labeling of carbohydrate and protein epitopes. Nat. Protoc., 7(9):17161727, August 2012.

[3] R. D. Allen, G. B. David, and G. Nomarski. The Zeiss-Nomarski differential interference equipment for transmitted-light microscopy. Z. Wiss. Mikrosk., 69(4):193-221, 1969.

[4] Y. Shechtman, Y. C. Eldar, O. Cohen, H. N. Chapman, J. Miao, and M. Segev. Phase retrieval with application to optical imaging. IEEE Signal Process. Mag., 32(3):87-109, 2015.

[5] E. B. van Munster, L. J. van Vliet, and J. A. Aten. Reconstruction of optical pathlength distributions from images obtained by a wide-field differential interference contrast microscope. J. Microsc., 188(2):149-157, 1997.

[6] Z. Kam. Microscopic differential interference contrast image processing by line integration (LID) and deconvolution. Bioimaging, 6(4):166-176, 1998.

[7] S. S. Kou, L. Waller, G. Barbastathis, and C. J. R. Sheppard. Transport-of-intensity approach to differential interference contrast (TI-DIC) microscopy for quantitative phase imaging. Opt. Lett., 35(3):447-449, 2010.

[8] E. Bostan, E. Froustey, B. Rappaz, E. Shaffer, D. Sage, and M. Unser. Phase retrieval by using transport-of-intensity equation and differential interference contrast microscopy. In Proc. 21th IEEE International Conference on Image Processing, pages 3939-3943, 2014.

[9] C. Preza, D. L. Snyder, and J.-A. Conchello. Image reconstruction for three-dimensional transmitted-light DIC microscopy. In J.-A. Conchello, C. J. Cogswell, and T. Wilson, editors, Three-Dimensional Microscopy: Image Acquisition and Processing IV, volume 2984 of Proc. SPIE, pages 220-231, 1997.

[10] C. Preza, D. L. Snyder, and J.-A. Conchello. Theoretical development and experimental evaluation of imaging models for differential interference contrast microscopy. J. Opt. Soc. Am. A, 16(9):2185-2199, 1999.

[11] C. Preza. Rotational-diversity phase estimation from differential interference contrast microscopy images. J. Opt. Soc. Am. A, 17(3):415-424, 2000.

[12] C. Preza, S. V. King, and C. J. Cogswell. Algorithms for extracting true phase from rotationallydiverse and phase-shifted DIC images. In J.-A. Conchello, C. J. Cogswell, and T. Wilson, editors, 
Three-Dimensional and Multidimensional Microscopy: Image Acquisition and Processing XIII, volume 6090 of Proc. SPIE, 2006.

[13] P. Charbonnier, L. Blanc-Féraud, G. Aubert, and M. Barlaud. Deterministic edge-preserving regularization in computed imaging. IEEE Trans. Image Process., 6(2):298-311, February 1997.

[14] S. Bonettini, I. Loris, F. Porta, and M. Prato. Variable metric inexact line-search based methods for nonsmooth optimization. SIAM J. Optim., 26(2):891-921, 2016.

[15] D. B. Murphy. Fundamentals of light microscopy and electronic imaging. Wiley-Liss, New York, 2001.

[16] S. B. Mehta and C. J. R. Sheppard. Partially coherent image formation in differential interference contrast (DIC) microscope. Opt. Express, 16(24):19462-19479, November 2008.

[17] L. Bautista, S. Rebegoldi, L. Blanc-Féraud, M. Prato, L. Zanni, and A. Plata. Phase estimation in differential-interference-contrast (DIC) microscopy. In Proc. IEEE Int. Symp. Biomed. Imaging, pages 136-139, 2016.

[18] J. W. Goodman. Statistical Optics. Wiley, New York, 1984.

[19] S. Krantz and H. R. Parks. A primer of Real Analytic Functions. Birkhäuser, 2002.

[20] R. Acar and C. R. Vogel. Analysis of bounded variation penalty methods for ill-posed problems. Inverse Probl., 10(6):1217-1229, June 1994.

[21] M. Bertero, P. Boccacci, G. Talenti, R. Zanella, and L. Zanni. A discrepancy principle for Poisson data. Inverse Probl., 26(10):105004, October 2010.

[22] L. I. Rudin, S. Osher, and E. Fatemi. Nonlinear total variation based noise removal algorithms. J. Phys. D., 60(1-4):259-268, 1992.

[23] J. B. Hiriart-Urruty and C. Lemarchal. Convex analysis and minimization algorithms. I. SpringerVerlag, Berlin, 1993.

[24] J. Nocedal and S. J. Wright. Numerical optimization. Springer, New York, 2nd edition, 2006.

[25] R. Fletcher. A limited memory steepest descent method. Math. Program., 135(1-2):413-436, October 2012.

[26] J. Barzilai and J. M. Borwein. Two-point step size gradient methods. IMA J. Numer. Anal., 8(1):141-148, January 1988.

[27] Y. H. Dai and Y. X. Yuan. Alternate minimization gradient method. IMA J. Numer. Anal., 23(3):377-393, July 2003.

[28] G. Frassoldati, G. Zanghirati, and L. Zanni. New adaptive stepsize selections in gradient methods. J. Ind. Manage. Optim., 4(2):299-312, May 2008.

[29] B. Zhou, L. Gao, and Y. H. Dai. Gradient methods with adaptive step-sizes. Comput. Optim. Appl., 35(1):69-86, September 2006.

[30] S. Bonettini, R. Zanella, and L. Zanni. A scaled gradient projection method for constrained image deblurring. Inverse Probl., 25(1):015002, January 2009.

[31] S. Łojasiewicz. Une propriété topologique des sous-ensembles analytiques réels. In Les Équations aux Dérivées Partielles, pages 87-89. Éditions du Centre National de la Recherche Scientifique, Paris, 1963.

[32] K. Kurdyka. On gradients of functions definable in o-minimal structures. Ann. Inst. Fourier, 48(3):769-783, 1998.

[33] H. Attouch, J. Bolte, and B. F. Svaiter. Convergence of descent methods for semi-algebraic and tame problems: proximal algorithms, forward-backward splitting, and regularized Gauss-Seidel methods. Math. Program., 137(1-2):91-129, February 2013.

[34] P. Frankel, G. Garrigos, and J. Peypouquet. Splitting methods with variable metric for KurdykaLojasiewicz functions and general convergence rates. J. Optim. Theory Appl., 165(3):874-900, June 2015.

[35] S. Bonettini, I. Loris, F. Porta, M. Prato, and S. Rebegoldi. On the convergence of a linesearch based proximal-gradient method for nonconvex optimization. Inverse Probl., 33:055005, 2017.

[36] Y. Xu and W. Yin. A block coordinate descent method for regularized multiconvex optimization with applications to nonnegative tensor factorization and completion. SIAM J. Imaging Sci., 
6(3):1758-1789, 2013.

[37] H. Lantéri, M. Roche, and C. Aime. Penalized maximum likelihood image restoration with positivity constraints: multiplicative algorithms. Inverse Probl., 18(5):1397-1419, October 2002.

[38] S. Bonettini, G. Landi, E. Loli Piccolomini, and L. Zanni. Scaling techniques for gradient projection-type methods in astronomical image deblurring. Int. J. Comput. Math., 90(1):929, January 2013.

[39] S. Bonettini, A. Chiuso, and M. Prato. A scaled gradient projection method for Bayesian learning in dynamical systems. SIAM J. Sci. Comput., 37(3):A1297-A1318, 2015.

[40] R. Fletcher. Practical methods of optimization. John Wiley and Sons, New York, 2nd edition, 2000.

[41] J. C. Gilbert and J. Nocedal. Global convergence properties of conjugate gradient methods for optimization. SIAM J. Optim., 2(1):21-42, 1992.

[42] M. Prato, R. Cavicchioli, L. Zanni, P. Boccacci, and M. Bertero. Efficient deconvolution methods for astronomical imaging: algorithms and IDL-GPU codes. Astron. Astrophys., 539:A133, March 2012.

[43] A. Beck and M. Teboulle. A fast iterative shrinkage-thresholding algorithm for linear inverse problems. SIAM J. Imaging Sci., 2(1):183-202, 2009.

[44] Bruker AFM Probes. Product description APCS-0099. http://www.brukerafmprobes.com/ a-3472-apcs-0099. aspx, July 2016.

[45] F. Porta, R. Zanella, G. Zanghirati, and L. Zanni. Limited-memory scaled gradient projection methods for real-time image deconvolution in microscopy. Commun. Nonlinear Sci. Numer. Simul., 21(1-3):112-127, April 2015.

[46] F. Porta, M. Prato, and L. Zanni. A new steplength selection for scaled gradient methods with application to image deblurring. J. Sci. Comput., 65(3):895-919, December 2015. 